\title{
Black holes in supergravity and integrability
}

\author{
W. Chemissany, ${ }^{a}$ P. Fré, ${ }^{b, c}$ J. Rosseel, ${ }^{d}$ A.S. Sorin, ${ }^{e}$ M. Trigiante $^{f}$ and T. Van Riet ${ }^{g}$ \\ ${ }^{a}$ University of Lethbridge, Physics Dept., \\ Lethbridge T1K 3M4, Alberta, Canada \\ ${ }^{b}$ Dipartimento di Fisica Teorica, Università di Torino, \\ via P. Giuria 1, I-10125 Torino, Italy \\ ${ }^{c}$ INFN, Sezione di Torino, \\ via P. Giuria 1, I-10125 Torino, Italy \\ ${ }^{d}$ Centre for Theoretical Physics, University of Groningen, \\ Nijenborgh 4, 9747 AG Groningen, The Netherlands \\ ${ }^{e}$ Bogoliubov Laboratory of Theoretical Physics, Joint Institute for Nuclear Research, \\ 141980 Dubna, Moscow Region, Russia \\ ${ }^{f}$ Dipartimento di Fisica Politecnico di Torino, \\ C.so Duca degli Abruzzi, 24, I-10129 Torino, Italy \\ ${ }^{g}$ Institutionen för Fysik och Astronomi, \\ Box 803, SE-751 08 Uppsala, Sweden \\ E-mail: wissam.chemissany@uleth.ca, fre@to.infn.it, j.Rosseel@rug.nl, \\ sorin@theor.jinr.ru, mario.trigiante@polito.it, \\ thomas.vanriet@fysast.uu.se
}

ABSTRACT: Stationary black holes of massless supergravity theories are described by certain geodesic curves on the target space that is obtained after dimensional reduction over time. When the target space is a symmetric coset space we make use of the grouptheoretical structure to prove that the second order geodesic equations are integrable in the sense of Liouville, by explicitly constructing the correct amount of Hamiltonians in involution. This implies that the Hamilton-Jacobi formalism can be applied, which proves that all such black hole solutions, including non-extremal solutions, possess a description in terms of a (fake) superpotential. Furthermore, we improve the existing integration method by the construction of a Lax integration algorithm that integrates the second order equations in one step instead of the usual two step procedure. We illustrate this technology with a specific example

KEYwords: p-branes, Black Holes in String Theory, D-branes, Global Symmetries ARXIV EPRINT: 1007.3209 


\section{Contents}

1 Introduction 2

2 Geodesic curves on symmetric spaces 4

2.1 Symmetric spaces 4

2.2 Geodesic Lax equations 6

$\begin{array}{lll}2.3 & \text { Noether charges } & 7\end{array}$

$\begin{array}{llr}3 & \text { Integrability } & \mathbf{8}\end{array}$

3.1 Preliminary facts 8

$\begin{array}{ll}3.2 & \text { The proof of Liouville integrability } \\ \end{array}$

3.3 From Liouville to Hamilton-Jacobi and (fake) superpotentials 11

4 Lax integration algorithms $\quad 14$

$\begin{array}{lll}4.1 & \text { Integration formula for the Lax operator } & 15\end{array}$

$\begin{array}{lll}4.2 & \text { An integration formula for the coset representative } & 16\end{array}$

$\begin{array}{lll}5 & \text { Applications to black holes } & 17\end{array}$

$\begin{array}{lll}5.1 & \text { Black holes as geodesics } & 17\end{array}$

$\begin{array}{lll}5.2 & \text { The structure of } \mathfrak{g} & 20\end{array}$

5.3 The solvable parametrization and the Noether charges 22

5.4 The (fake) superpotentials $\mathcal{W}_{4}, \mathcal{W}_{3}$

$\begin{array}{ll}5.5 & \text { The hamiltonians } \\ 5.6 & 25\end{array}$

$\begin{array}{lll}5.6 & \text { Regularity } 25\end{array}$

$\begin{array}{lll}5.7 & \text { Normal forms } & 27\end{array}$

5.8 The dilatonic black hole 28

5.8.1 The $\frac{\mathrm{S} \ell(3, \mathbb{R})}{\mathrm{SO}(2,1)}$ sigma model 28

$\begin{array}{lll}5.8 .2 & \text { The solutions } & 29\end{array}$

$\begin{array}{ll}\text { 5.8.3 The normal form } & 30\end{array}$

5.8.4 Hamiltonian viewpoint 31

$\begin{array}{lll}6 & \text { Discussion } & 33\end{array}$

A The sigma model in $d=3+0 \quad 35$

$\begin{array}{ll}\text { B Conventions for charges and mass } & 36\end{array}$ 


\section{Introduction}

The study and construction of black hole solutions in supergravity has a long history [1-5]. A prominent role in this history is played by solutions that preserve some supersymmetry. Such solutions are easier to construct because the preservation of some fraction of supersymmetry leads to first order equations instead of the usual second order equations of motion. Furthermore, supersymmetric solutions are protected, to some extent, from quantum corrections and this allowed to compare supergravity states with open string states, and thus to verify the celebrated Bekenstein-Hawking law for the black hole entropy from direct microstate counting.

However, non-supersymmetric solutions are perhaps even more interesting. The lack of first order BPS equations, that arise from demanding preservation of supersymmetry, implies that we need to find other tools to integrate the second order equations of motion in this case. This paper is concerned with providing such tools. Our analysis will be restricted to spherically symmetric, asymptotically flat black holes.

The second order differential equations can be derived from two different, but equivalent, effective actions and the tools that are developed for studying non-supersymmetric solutions depend on the choice of the effective action. Let us briefly summarize these matters, assuming the simplest case of stationary, spherically symmetric (single-centered) black holes. We shall be mainly dealing with static solutions in four dimensions, although our analysis in three dimensions will allow us to extend our analysis by introducing a TaubNUT charge. Furthermore we consider four-dimensional solutions, the extension to any other dimension being straightforward.

The first kind of effective action is obtained, for static solutions, by expressing the Maxwell field strengths in terms of the quantized magnetic and electric charges $m^{I}$ and $e_{I}$ via the respective equations of motion (and Bianchi identities). Then all remaining degrees of freedom depend on the radial coordinate $\tau$, rendering the effective action onedimensional. The action thus obtained describes the motion of a particle subject to an external force field described by the effective, non-positive, potential $-V(\phi, m, e)[6,7]$

$$
\mathcal{L}=\dot{U}^{2}+\frac{1}{2} G_{r s} \dot{\phi}^{r} \dot{\phi}^{s}+\mathrm{e}^{2 U} V\left(\phi^{r}, m, e\right),
$$

where $V \geq 0$ is called the effective black hole potential and $e^{2 U}$ is the time-component of the static metric. The $\phi^{r}$-dependence of $V$ arises from the coupling of the scalars to the vectors and where we have used $\dot{\phi}^{r}$ to denote the derivative of the fields with respect to the radial variable $\tau$. In this framework the main tool to describe non-supersymmetric extremal solutions is by mimicking the supersymmetric case. This means that one tries to find a function $\mathcal{W}_{4}\left(U, \phi^{r} ; m, e\right)$, named the "fake superpotential" or "fake central charge", such that $2 \mathrm{e}^{2 U} V=\frac{1}{2}\left(\partial_{U} \mathcal{W}_{4}\right)^{2}+\partial_{r} \mathcal{W}_{4} \partial^{r} \mathcal{W}_{4}$. If this situation is realized the action can be rewritten as a sum (and difference) of squares. Putting the separate squares to zero leads to first order flow equations

$$
\dot{\phi}^{r}=G^{r s} \partial_{s} \mathcal{W}_{4}, \quad \dot{U}=\partial_{U} \mathcal{W}_{4} .
$$

This is nothing else but the familiar Hamilton-Jacobi integration method [8], and $\mathcal{W}_{4}$ is the Hamilton's characteristic function associated with the autonomous system (1.1). Such 
road to the understanding of non-susy extremal solutions has been used in e.g. [9-15] and references therein. It has later been realized, starting with [16], that this approach could be extended to the non-extremal Reissner-Nordström black hole, and it has subsequently been generalized to other, more involved, non-extremal solutions [17-19]. ${ }^{1}$

In this paper we use the second form of the effective action, found in [20], which is obtained by dimensional reduction of the four-dimensional action over the timelike direction to three dimensions. In three dimensions all vectors can be dualised to scalars such that one ends up with a supergravity theory, whose bosonic part of the action is given by

$$
S=\int \mathrm{d}^{3} x \sqrt{g_{3}}\left(\frac{1}{2} R_{3}-\frac{1}{2} g_{i j}(\phi) \partial \phi^{i} \partial \phi^{j}\right) .
$$

The scalars $\phi^{i}$ parametrize a target space with metric $g_{i j}(\phi)$ of indefinite signature [21-24]. One can show that gravity decouples from the sigma model such that the non-trivial part of the effective action becomes a pure geodesic problem

$$
\mathcal{L}=\frac{1}{2} g_{i j} \dot{\phi}^{i} \dot{\phi}^{j}
$$

The target space in three dimensions (with metric $g_{i j}$ ) contains the target space in four dimensions (with metric $G_{r s}$ ) as a subspace. This description of supergravity solutions as geodesic curves goes beyond four-dimensional black holes and works for generic supergravity solutions that depend effectively on one direction, like e.g. stationary and cosmological $p$ brane solutions, wormholes, instantons, and so on (see [25] and references therein).

Similar to the first effective action (1.1), the Hamilton-Jacobi formalism, if it can be applied, leads to a (fake) superpotential $\mathcal{W}_{3}\left(\phi^{i}\right)$ such that the second order differential equations can be integrated to first order flow equations

$$
\dot{\phi}^{i}=g^{i j} \partial_{j} \mathcal{W}_{3} .
$$

As explained in $[8], \mathcal{W}_{4}\left(U, \phi^{r} ; m, e\right)$ is related to $\mathcal{W}_{3}\left(\phi^{i}\right)$ in a simple manner. We shall mainly be concerned with the latter, which we shall simply denote by $\mathcal{W}$.

Both approaches (1.1)nd (1.4) are equivalent, but the second approach (1.4) is beneficial since it makes more symmetries apparent. Moreover, when the target space in three dimensions is a symmetric space (as is often the case in extended supergravities), ${ }^{2}$ one can use group theoretical algorithms to integrate the second order equations of motion, see e.g. $[20,25-47]$.

These algorithms were first established for the case that the target space is Riemannian. This case is relevant for the construction of cosmological solutions in supergravity [34, 35, 39, 42]. Later developments have generalized the integration procedures to target spaces of indefinite signature [37, 40, 41, 48], that find applications in describing stationary solutions such as black holes. Essentially, these algorithms solve the geodesic equations in a two step process. In the first step, one relies on the fact that the geodesic equations can be

\footnotetext{
${ }^{1}$ The most general form of the non-extremal flow equations were derived in [19].

${ }^{2}$ The target spaces in supergravity are symmetric for theories with more than 8 supercharges. An interesting subset of theories with 8 or less supercharges exhibits symmetric target spaces as well.
} 
rewritten as a Lax pair equation, i.e. as a first order matrix differential equation for the tangent velocities. In the second step, one eventually solves the first order system defined by the expression for the tangent vector.

The fact that the geodesic equations on symmetric spaces can be written in a Lax pair form, is a strong indication that these equations are Liouville integrable, since this is a necessary condition for Liouville integrability [49]. In a recent paper, [48] the Liouville integrability of the first order equation for the tangent vector was proven, whereas in this paper we establish the Liouville integrability of the full second order geodesic equations. This we prove by explicitly constructing the correct number of conserved quantities (a.k.a Hamiltonians) that mutually Poisson-commute (a.k.a are in involution). These involutive conserved quantities are appropriate non trivial rational functions of the Noether charges. The explicit form of the involutive hamiltonians was discussed in [48] for the case of $\mathrm{S} \ell(N, \mathbb{R})$ algebras (such construction can be shown to work for a variety of other symmetric geometries such as the STU model). Its general form will be presented in a forthcoming paper [50]. To our knowledge such constructive proof of Liouville integrability for this class of models is not present in the existing literature.

Liouville integrability implies that there always exists a description in terms of a (fake) superpotential. This (fake) superpotential can be lifted to the (fake) superpotential in the usual four-dimensional formulation that uses a black hole effective potential [8]. Therefore, in this paper we prove that all spherically symmetric solutions of symmetric supergravity theories where the scalar manifold is a homogeneous coset allow a (fake) superpotential. ${ }^{3}$ This result is not only important for the understanding of the differential equations that govern black hole solutions, but the existence of a fake superpotential, in the extremal case, comes with specific implications for the physics of black holes. In particular there are implications for the asymptotic stability of the attractor points (for large and small black holes) [51]. There are also implications in the context of holography [52, 53].

As suggested by Liouville integrability, we find a novel way to directly integrate the geodesic equations of motion in a one-step procedure to find the expression for the coset element. This is a significant improvement of the existing integration algorithms.

This paper is organised as follows. In section 2 we present the basic mathematical notions of symmetric spaces and geodesic equations in Lax pair form. In section 3 the proof of Liouville integrability of the second order geodesic equations is presented. Section 4, on the other hand deals with the practical integration procedure of the geodesic equations. Finally, in section 5 we illustrate and apply this technology to black hole solutions in simple supergravity theories. We conclude with a discussion in section 6 .

\section{Geodesic curves on symmetric spaces}

\subsection{Symmetric spaces}

Consider a real $n$-dimensional symmetric space $G / H^{*}$, where $G$ is a semisimple Lie algebra and $H^{*}$ is a maximal subgroup. For pseudo-Riemannian manifolds, $H^{*}$ is a non-compact

\footnotetext{
${ }^{3}$ This has been conjectured in [18].
} 
real form of some complex semisimple Lie group, whose compact real form will be denoted by $H$. We denote the Lie algebras of $G$ and $H^{*}$ by $\mathfrak{g}, \mathfrak{H}^{*}$ respectively. The orthogonal ${ }^{4}$ complement to $\mathfrak{H}^{*}$ in $\mathfrak{g}$ is denoted by $\mathfrak{K}$

$$
\mathfrak{g}=\mathfrak{H}^{*} \oplus \mathfrak{K} .
$$

This decomposition is called the Cartan decomposition and alternatively it can be defined by introducing an involutive automorphism $\theta$, the so-called Cartan involution. This Cartan involution acts as follows

$$
\theta\left(\mathfrak{H}^{*}\right)=\mathfrak{H}^{*}, \quad \theta(\mathfrak{K})=-\mathfrak{K} .
$$

As $\theta$ preserves the Lie brackets, we obtain

$$
\left[\mathfrak{H}^{*}, \mathfrak{H}^{*}\right] \subset \mathfrak{H}^{*}, \quad[\mathfrak{K}, \mathfrak{K}] \subset \mathfrak{H}^{*}, \quad\left[\mathfrak{H}^{*}, \mathfrak{K}\right] \subset \mathfrak{K} .
$$

In case one works in a real matrix representation, the action of $\theta$ can be expressed in terms of a diagonal matrix $\eta$ (see e.g. [25]):

$$
\forall X \in \mathfrak{g}: \theta(X)=-\eta X^{T} \eta, \quad \eta=\operatorname{diag}\left(\mathbb{1}_{p},-\mathbb{1}_{q}\right) .
$$

Let us also introduce a different decomposition of $\mathfrak{g}$ that will be useful throughout this article. This is the so-called Iwasawa decomposition of $\mathfrak{g}$

$$
\mathfrak{g}=\mathfrak{H}^{*} \oplus S o l v,
$$

where $S o l v$ is a maximal solvable subalgebra of $\mathfrak{g}$. The corresponding decomposition at the level of group elements does not hold globally on $G / H^{*}$, since not all elements $g$ of $G$ can be expressed in the form $g=s \cdot h^{*}$, where $s \in \exp (S o l v)$ is a solvable group element and $h^{*} \in H^{*}$.

We denote the generators of the solvable algebra by $T_{A},{ }^{5}$ the generators of $\mathfrak{H}^{*}$ by $H_{\alpha}$ and the generators of $\mathfrak{K}$ by $K_{A}$. The following relations then hold

$$
\begin{aligned}
K_{A} & =\frac{1}{2}\left(T_{A}+\eta T_{A}^{T} \eta\right), \\
H_{\alpha} & =\left\{\text { Non-vanishing combinations } \frac{1}{2}\left(T_{A}-\eta T_{A}^{T} \eta\right)\right\} .
\end{aligned}
$$

Choosing a parametrization on $G / H^{*}$ amounts to defining a coset representative $\mathbb{L}(\phi) \in$ $G / H^{*}$. It is useful to choose this representative in the so-called solvable gauge, meaning that it is obtained by exponentiating a generic element of the solvable algebra Solv of (2.5)

$$
\mathbb{L}(\phi)=\exp \left(\phi^{i} T_{i}\right),
$$

where we have used the index $i$ to label the $3 d$ scalar fields and consequently also used that index for the solvable generators here. Since, as previously mentioned, the Iwasawa

\footnotetext{
${ }^{4}$ For symmetric spaces $G$ is semi-simple and we define 'orthogonal' with respect to the Cartan-Killing metric of $\mathfrak{g}$.

${ }^{5}$ Or sometimes by $T_{i}$.
} 
decomposition of $G$ with respect to $H^{*}$ holds only locally in $G / H^{*}$, the solvable coordinates $\phi^{i}$ do not provide a set of global coordinates but only span a patch of the whole manifold. However this is not an issue for black hole solutions as we explain in section 5.3.

For the time being, we restrict ourselves to the solvable patch of $G / H^{*}$, which is isometric to the solvable group manifold $\exp (S o l v)$, the metric being defined below. The vielbein and connection 1-forms, $\mathrm{d} \phi^{i} V_{i}{ }^{A}$ and $\mathrm{d} \phi^{i} \mathcal{W}_{i}{ }^{\alpha}$ resp., are defined via the decomposition

$$
\mathbb{L}^{-1} \mathrm{~d} \mathbb{L}=\mathrm{d} \phi^{i} V_{i}^{A} T_{A}=\mathrm{d} \phi^{i} V_{i}^{A} K_{A}+\mathrm{d} \phi^{i} \mathcal{W}_{i}^{\alpha} H_{\alpha} .
$$

The metric $g_{A B}$ on the tangent space to the manifold at the origin is proportional to the restriction of the Cartan-Killing metric on $\mathfrak{g}$ to $\mathfrak{K}$

$$
g_{i j}=g_{A B} V_{i}^{A} V_{j}^{B}, \quad g_{A B}=\alpha \tilde{\eta}_{A B}, \quad \tilde{\eta}_{A B} \equiv \operatorname{Tr}\left(K_{A} K_{B}\right),
$$

where $V_{i}{ }^{A}$ is the vielbein matrix. The normalization factor $\alpha$ depends on the representation of the generators $K_{A}$ and is chosen so as to have the standard normalization for the kinetic terms of the scalars in the Lagrangian. The algebraic structure of Solv is encoded in its structure constants $f_{A B}{ }^{C}$ and can be either described by the Maurer-Cartan equations for the 1-forms $V^{A} \equiv \mathrm{d} \phi^{i} V_{i}{ }^{A}$ or by the commutation relations among the generators $T_{A}$

$$
\mathrm{d} V^{A}=-\frac{1}{2} f_{B C}{ }^{A} V^{B} \wedge V^{C},\left[T_{A}, T_{B}\right]=f_{A B}{ }^{C} T_{C} .
$$

In view of the following analysis it is useful to define the adjoint representation on Solv of the coset representative $\mathbb{L}\left(\phi^{i}\right) \in \exp (S o l v)$ and of each single generator $T_{A}$

$$
\mathbb{L}^{-1} T_{A} \mathbb{L}=\mathbb{L}_{A}^{B} T_{B}, \quad\left(T_{A}\right)_{B}^{C}=-f_{A B}{ }^{C} .
$$

Then the first equality in (2.9) can be written in the adjoint representation as follows

$$
\left(\mathbb{L}^{-1}\right)_{B}{ }^{D} \partial_{i} \mathbb{L}_{D}{ }^{C}=-V_{i}^{A} f_{A B}^{C},
$$

where $\partial_{i} \equiv \frac{\partial}{\partial \phi^{2}}$.

\subsection{Geodesic Lax equations}

Let us now discuss the general features of geodesics on $G / H^{*}$ and their description in terms of a Lax pair equation. Consider a geodesic on $G / H^{*}$ defined by $n$-functions $\phi^{i}(\tau)$ of an affine parameter $\tau$. In analogy with the Riemannian case [43], it was established in $[40,41,48]$ that one can rewrite the geodesic equations on a symmetric space of indefinite signature as a matrix differential equation of the Lax form

$$
\frac{\mathrm{d}}{\mathrm{d} \tau} L=[L, W]
$$

The Lax operator $L$ and Lax connection $W$ are defined in terms of the pull-back on the geodesic of the left-invariant 1 -form on $G / H^{*}$, which we denote by $\Omega$

$$
\begin{aligned}
\Omega & \equiv \mathbb{L}^{-1} \frac{\mathrm{d}}{\mathrm{d} \tau} \mathbb{L}=\dot{\phi}^{i} \mathbb{L}^{-1} \frac{\partial}{\partial \phi^{i}} \mathbb{L}=Y^{A} T_{A}=W+L, \\
L & =Y^{A} K_{A}, \\
Y^{A} & =\tilde{\eta}^{A B} \operatorname{Tr}\left(\Omega K_{B}\right),
\end{aligned}
$$


where $\tilde{\eta}^{A B}$ denotes the inverse of $\tilde{\eta}_{A B}$. From the above definition it follows that the components $Y^{A}$ of the Lax matrix $L$ are the pull-back on the geodesic of the vielbein 1-forms

$$
Y^{A} \equiv \dot{\phi}^{i} V_{i}^{A}
$$

Note that $W$ and $L$ depend on the scalars $\phi$ as well as on their derivatives $\dot{\phi}$ with respect to $\tau$. Then the geodesic action can be written as

$$
S=\int \mathrm{d} \tau \mathcal{L} \equiv \int \mathrm{d} \tau \frac{1}{2} g_{A B} Y^{A} Y^{B}=\int \mathrm{d} \tau \frac{\alpha}{2} \operatorname{Tr}(L L)
$$

where we have used (2.10). When one works in the solvable gauge, one can choose a representation for which the Lax connection $W$ is given in terms of $L$ as follows

$$
W=L_{>}-L_{<}
$$

where $L_{>(<)}$denotes the upper-triangular (resp. lower-triangular) part of $L$. We are therefore interested in solving the following Lax equation

$$
\frac{\mathrm{d}}{\mathrm{d} \tau} L+\left[L_{>}-L_{<}, L\right]=0
$$

Algorithmic methods to achieve this have been devised in the mathematical and physical literature $[37,40,41,43,48,54-56]$. The integration formulae developed, allow one to obtain an explicit solution $L_{\mathrm{sol}}(\tau)$ of $(2.20)$, obeying the initial condition $L_{\mathrm{sol}}(\tau=0)=L_{0}$. In order to extract the solutions for the scalar fields, one should still solve the system of differential equations obtained from

$$
\sum_{A} \operatorname{Tr}\left(\mathbb{L}(\phi(\tau))^{-1} \frac{\mathrm{d}}{\mathrm{d} \tau} \mathbb{L}(\phi(\tau)) K_{A}\right) \tilde{\eta}^{A B} K_{B}=L_{\mathrm{sol}}(\tau)
$$

It turns out that, thanks to the use of the solvable gauge, this system of differential equations can be solved iteratively [34].

The fact that the geodesic equations can be written in a Lax pair form is therefore a strong hint of the integrability of the black hole equations of motion, viewed as geodesic equations. We should however keep in mind that the Lax equations are first order, while the geodesic equations are second order. Only the first integration step has been shown to be integrable [48]. The full integration process of the second order equations involves two steps and integrability of the first step does not yet establish integrability of the full system.

In section 3, we give a proof of Liouville integrability of the full second order geodesic equations. In section 4, we give a novel, more practical, way to obtain solutions of the geodesic equations.

\section{$2.3 \quad$ Noether charges}

Consider the following matrix

$$
Q=\mathbb{L}(\tau) L(\tau) \mathbb{L}(\tau)^{-1}
$$


Using the Lax equation one can infer that $Q$ is a constant of motion: $\frac{\mathrm{d} Q}{\mathrm{~d} \tau}=0$. In fact, $Q$ is the matrix of Noether charges and plays an important role in establishing Liouville integrability. While $L$ is an element of $\mathfrak{K}, Q$ is an element of the whole algebra $\mathfrak{g}$.

There is an alternative method to describe the geodesic action in terms of the symmetric coset matrix

$$
\mathbb{M}(\phi)=\mathbb{L}(\phi) \eta \mathbb{L}(\phi)^{T},
$$

where $\eta$ was introduced in (2.4). The geodesic action then reads

$$
S=-\frac{\alpha}{8} \int \mathrm{d} \tau \operatorname{Tr}\left(\frac{\mathrm{d}}{\mathrm{d} \tau} \mathbb{M} \frac{\mathrm{d}}{\mathrm{d} \tau} \mathbb{M}^{-1}\right) .
$$

In this language the solution is immediate (see e.g. [25])

$$
\mathbb{M}(\tau) \equiv \mathbb{M}\left(\phi^{i}(\tau)\right)=\mathbb{M}(0) \mathrm{e}^{2 Q^{T} \tau},
$$

where $\mathbb{M}(0)$ is the matrix $\mathbb{M}$ computed on the values $\phi^{i}(0)$ of the scalar fields $\phi^{i}$ at $\tau=0$. The geodesic is thus uniquely defined by the matrix $Q$ and the initial point $\phi^{i}(0)$ on the manifold. From definition (2.22) it follows that $\mathbb{M}(0) Q^{T}=Q \mathbb{M}(0)$. On the other hand, by definition, $\mathbb{M}(\tau)$ should be symmetric for all $\tau$ and the considered solution implements such property. When the initial condition is chosen at the origin, $\mathbb{M}(0)=\eta$, we find that $Q \in \mathfrak{K}$

$$
Q^{T}=\eta Q \eta \text {. }
$$

For generic initial conditions $Q$ is an element of a subspace of $\mathfrak{g}$ which is isomorphic to $\mathfrak{K}$. In what follows we choose the moduli such that $\mathbb{M}(0)=\eta$.

Equation (2.25) is an explicit integration of the second order equations, however, not a useful one for matrices with dimension larger than two as the problem of extracting the individual scalar fields from the solution for $\mathbb{M}$ becomes non-trivial. Below we present an integration procedure that gives the explicit solution for the coset representative $\mathbb{L}$. This can then be used to find the profile of the individual scalars. The latter procedure is simpler than extracting the scalars out of $\mathbb{M}$ because of the upper-triangular structure of $\mathbb{L}$. The integration procedure we present below for $\mathbb{L}$ is related to the known Lax integration algorithms.

Fortunately, not all questions of black hole physics require the exact profile of the various scalar fields such that one can learn already various things in this framework. For instance, it is useful to understand the space of black hole solutions and its various subspaces, such as 1) the space of all regular solutions, 2) the space of supersymmetric solutions, 3) the space of extremal, regular non-supersymmetric solutions, and so on. It turns out that these spaces can all be characterized by simple constraints on $Q$ as we explain in section 5 .

\section{Integrability}

\subsection{Preliminary facts}

Consider the geodesic Lagrangian defined in (2.18)

$$
\mathcal{L}=\frac{1}{2} g_{A B} Y^{A} Y^{B}=\frac{1}{2} g_{i j} \dot{\phi}^{i} \dot{\phi}^{j} .
$$


Note that the above Lagrangian describes an autonomous system in which the radial variable $\tau$ plays the role of time. We define the momenta $P_{i}$ conjugate to $\phi^{i}$ and the Hamiltonian function

$$
P_{i} \equiv \frac{\partial \mathcal{L}}{\partial \dot{\phi}^{i}}=g_{i j} \dot{\phi}^{j}, \quad \mathcal{H}=\frac{1}{2} g^{i j} P_{i} P_{j}=\mathcal{L}
$$

The variables $\phi^{i}, P_{j}$ span the phase space of the system and will be collectively denoted $Z=\left\{\phi^{i}, P_{j}\right\}$. The Poisson bracket on phase space is defined as usual

$$
\left\{\phi^{i}, \phi^{j}\right\}=0, \quad\left\{P_{i}, P_{j}\right\}=0 \quad\left\{P_{i}, \phi^{j}\right\}=-\delta_{i}^{j} .
$$

We can write the geodesic equations of motion in a compact form as follows

$$
\dot{Z}+\{\mathcal{H}, Z\}=0
$$

From their definition (2.17) we derive the expression of $Y^{A}$ in terms of the conjugate variables

$$
Y^{A}=g^{A B} V_{B}^{i} P_{i}
$$

where $V_{A}^{i}$ denotes the inverse vielbein matrix $\left(V_{A}^{i} V_{B}{ }^{j} g^{A B}=g^{i j}\right)$ of the solvable group manifold. From (3.3) and (3.5) and the Maurer-Cartan equations (2.11) one verifies the following Poisson brackets

$$
\left\{Y_{A}, Y_{B}\right\}=-f_{A B}^{C} Y_{C},\left\{F\left(\phi^{i}\right), Y_{A}\right\}=V_{A}^{i} \frac{\partial F}{\partial \phi^{i}},
$$

where we have defined $Y_{A} \equiv g_{A B} Y^{B}$, which can be viewed as a basis for the dual solvable Lie algebra Solv* ${ }^{*}$ This is the natural Poisson bracket induced by the algebra. ${ }^{6}$

Let us now define the following $n$ components of the Noether charge matrix $Q$

$$
Q_{A} \equiv \alpha \operatorname{Tr}\left(Q T_{A}\right) .
$$

From the definition (2.22) of $Q$ one can derive the following relation between $Q_{A}$ and $Y_{A}$

$$
Q_{A} \equiv \mathbb{L}_{A}^{B} Y_{B}
$$

Aside from being constants of motion, the above relation and eq. (3.6) imply that $Q_{A}$ are in involution with the $Y^{A}$

$$
\left\{Q_{A}, Y_{B}\right\}=0,
$$

and that, moreover, they satisfy the following Poisson relations

$$
\left\{Q_{A}, Q_{B}\right\}=f_{A B}^{C} Q_{C} .
$$

\footnotetext{
${ }^{6}$ For any given Lie algebra $\mathfrak{g}$ we can turn the dual Lie algebra $\mathfrak{g} *$ into a Poisson manifold since there exists a natural way to define the Poisson bracket. Consider two functions $F_{1}, F_{2}$ on $\mathfrak{g} *$ and take $Y \in \mathfrak{g} *$, then we define the bracket $\{.,$.$\} as \left\{F_{1}, F_{2}\right\}(Y) \equiv\left(\partial^{A} F_{1}\right)\left(\partial^{B} F_{2}\right) f_{A B}{ }^{C} Y_{C}$, with $\partial^{A} F \equiv \frac{\partial F}{\partial Y_{A}}$. This Poisson bracket indeed reproduces (3.6) if the representation is taken with the minus $\operatorname{sign} T_{A} \rightarrow-T_{A}$. This was used in [37] to prove Liouville integrability of the first order problem for the geodesic tangent velocity vector.
} 
Hence, whereas the $Y_{A}$ are related to the Killing vectors associated with the right action of Solv, the $Q_{A}$ are related to the left action [57].

The $Y_{A}$ and $Q_{A}$ introduced above depend on the phase space variables $Z$ via their definitions. In the next section, we show that one can construct a number of constants of motion that depend on $Y_{A}$ and $Q_{A}$. These constants Poisson-commute and one can find the correct number of them, so as to constitute a complete set of Hamiltonians as required by Liouville integrability.

\subsection{The proof of Liouville integrability}

Liouville integrability, see for instance [58], is the statement that there exist $n$ functionally independent constants of motion $\mathcal{H}_{i}(Z)$, here referred to as Hamiltonians, that Poissoncommute with each other

$$
\left\{\mathcal{H}_{i}, \mathcal{H}_{j}\right\}=0 .
$$

This statement implies the usual Hamilton-Jacobi formulation of integrability in terms of a (fake) superpotential $W$, which is the language used in the supergravity literature. This is explained below in subsection 3.3.

What has been established sofar in [48] is the Liouville integrability of the first order problem

$$
\dot{Y}_{A}+\left\{\mathcal{H}, Y_{A}\right\}=0 .
$$

Since the Poisson bracket on the dual Lie algebra $S o l v *$ (the space spanned by the $Y_{A}$ ) is degenerate, integrability implies the existence of a symplectic foliation for which the Hamiltonian flow is Liouville integrable on the symplectic leaves. Each leaf is nothing but the co-adjoint orbit of an element $\left(Y_{A}\right)$ of $S_{o l v} *$. Denoting the dimension of the coset by $n$ and the dimension of the leaves by $2 h_{O}$ (since the symplectic leaves are always even dimensional), we have by definition

$$
2 h_{O}=\operatorname{rank}\left(f_{A B}^{C} Y_{C}\right) .
$$

Reference [48] showed the existence of $\left(n-h_{O}\right)$ constants of motion in involution, where $h_{O}$ of them correspond to Hamiltonians in involution on the symplectic leaf and the remaining $n-2 h_{O}$ constants are referred to as Casimirs. A Casimir $\mathcal{H}\left(Y_{A}\right)$ is defined by the property

$$
\left\{\mathcal{H}\left(Y_{A}\right), Y_{B}\right\}=0, \quad \forall B=1, \ldots, n .
$$

The Casimirs define the foliation since the symplectic leaves are labeled by the values of a maximal set of functionally independent Casimir functions. For the moment we are not interested in the exact form and construction of these Hamiltonians; we simply assume their existence. The explicit expression for the Hamiltonians can be found in [48] and we recall the relevant formulas in section 5 , when we need them explicitly.

Let us denote the Hamiltonians on the leaves by $\mathcal{H}_{a}\left(Y_{A}\right)$, where $a=1, \ldots, h_{O}$ and the Casimirs by $\mathcal{H}_{\ell}\left(Y_{A}\right)$, where $\ell=1, \ldots, n-2 h_{O}$. If we use the identities (3.9) and (3.10) we find $2\left(n-h_{O}\right)$ constants of motion, which Poisson-commute

$$
\mathcal{H}_{a}\left(Y_{A}\right), \quad \mathcal{H}_{\ell}\left(Y_{A}\right), \quad \mathcal{H}_{a}\left(Q_{A}\right), \quad \mathcal{H}_{\ell}\left(Q_{A}\right)
$$


where the $\mathcal{H}_{a}\left(Q_{A}\right)$, resp. $\mathcal{H}_{\ell}\left(Q_{A}\right)$ are obtained by replacing $Y_{A}$ by $Q_{A}$ in $\mathcal{H}_{a}\left(Y_{A}\right), \mathcal{H}_{\ell}\left(Y_{A}\right)$. One can show that each $\mathcal{H}_{\ell}\left(Q_{A}\right)$ is itself a Casimir, namely it satisfies eq. (3.14) and can thus be expressed as a function of the $\mathcal{H}_{\ell}\left(Y_{A}\right)$. To prove this, consider a generic representative $\mathcal{H}_{\ell}\left(Q_{A}\right)$ of this set. By construction it satisfies the equations obtained by replacing $Y_{A} \rightarrow Q_{A}$ in (3.14)

$$
\frac{\partial \mathcal{H}_{\ell}}{\partial Q_{A}}\left(Q_{E}\right) f_{A B}^{C} Q_{C}=0, \quad \forall B=1, \ldots, n .
$$

Using the relation (3.8) and the invariance of $f_{A B}{ }^{C}$ under the action of $\mathbb{L} \in \exp (S o l v)$, we conclude that $\mathcal{H}_{\ell}\left(Q_{A}\right)=\mathcal{H}_{\ell}\left(\mathbb{L}_{A}{ }^{B} Y_{B}\right)$, as a function of $Y_{B}$, satisfy eq. (3.14), and thus correspond to Casimirs.

The remaining $\mathcal{H}_{a}\left(Q_{A}\right)$ are independent of the $\mathcal{H}_{a}\left(Y_{A}\right)$ and $\mathcal{H}_{\ell}\left(Y_{A}\right)$. This gives us a total of

$$
\left(n-h_{O}\right)+h_{O}=n,
$$

Hamiltonians in involution, thereby proving Liouville integrability of the second order problem. The Hamiltonians $\mathcal{H}_{i}$ of eq. (3.11) are thus explicitly constructed as $\left\{\mathcal{H}_{i}\right\}=$ $\left\{\mathcal{H}_{a}(Y), \mathcal{H}_{\ell}(Y), \mathcal{H}_{a}(Q)\right\}$.

\subsection{From Liouville to Hamilton-Jacobi and (fake) superpotentials}

In order to fill the gap between the classical mathematical language of Liouville integrability and the language adopted in the current supergravity literature on black hole solutions, it is convenient to recall a few basic definitions and concepts concerning the momentum map on symplectic manifolds.

Let $\mathcal{M}_{2 n}$ be a real even-dimensional manifold endowed with a closed non-degenerate two-form:

$$
\begin{aligned}
\Omega & =\Omega_{\alpha \beta}(Z) d Z^{\alpha} \wedge d Z^{\beta}, \\
d \Omega & =0
\end{aligned}
$$

where $Z^{\alpha}$ denote the $2 n$ coordinates in any given patch. The pair $\left(\mathcal{M}_{2 n}, \Omega\right)$ is named a symplectic manifold. Consider moreover vector-fields on the manifold $\mathcal{M}_{2 n}$

$$
\mathbf{X}=X^{\alpha} \frac{\partial}{\partial Z^{\alpha}},
$$

with the property that they respect the symplectic structure induced by $\Omega$. This means that the Lie-derivative of $\Omega$ along $\mathbf{X}$ vanishes:

$$
0=\ell_{\mathbf{X}} \Omega \equiv i_{\mathbf{X}} d \Omega+d i_{\mathbf{X}} \Omega
$$

In the above equation the symbol $i_{\mathbf{X}}$ denotes contraction of the given form along the mentioned vector field. Vector fields fulfilling eq. (3.20) are named symplectic. It follows from this definition that for any symplectic vector field $\mathbf{X}$ the one-form $i_{\mathbf{X}} \Omega$ is closed and 
hence locally exact on any open neighborhood $\mathcal{U} \subset \mathcal{M}_{2 n}$. In other words on any $\mathcal{U}$ we can construct a function $\mathfrak{P}_{\mathbf{X}}^{(\mathcal{U})}$ which solves the following equation:

$$
\left.i_{\mathbf{X}} \Omega\right|_{\mathcal{U}}=\left.d \mathfrak{P}_{\mathbf{X}}^{(\mathcal{U})}\right|_{\mathcal{U}}
$$

The map from the tensor product of the tangent space $T \mathcal{U}$ with $\mathcal{U}$ into the real numbers:

$$
\mathfrak{P}_{\mathrm{X}}^{(\mathcal{U})}: T \mathcal{U} \times \mathcal{U} \mapsto \mathbb{R}
$$

is named the momentum-map. Clearly the non-trivial topology of the symplectic manifold reflects itself into the fact that for the same vector field $\mathbf{X}$ the momentum-map on the intersection of different patches $\mathcal{U}$ and $\mathcal{U}^{\prime}$ can be related by a non-trivial transition function $f_{\mathcal{U} \mathcal{U}^{\prime}}: \mathcal{U} \cap \mathcal{U}^{\prime} \mapsto \mathbb{R}$. Namely we have:

$$
\left.\mathfrak{P}_{\mathbf{X}}^{(\mathcal{U})}\right|_{\mathcal{U} \cap \mathcal{U}^{\prime}}=\left.f_{\mathcal{U} \mathcal{U}^{\prime}} \mathfrak{P}_{\mathbf{X}}^{\left(\mathcal{U}^{\prime}\right)}\right|_{\mathcal{U} \cap \mathcal{U}^{\prime}}
$$

Given the existence of the momentum map, one can introduce the Poisson bracket of any two functions ${ }^{7} \mathfrak{P}_{\mathbf{X}}$ and $\mathfrak{P}_{\mathbf{Y}}$ associated with two different symplectic vector fields $\mathbf{X}$ and $\mathbf{Y}$ by means of the following:

$$
\left\{\mathfrak{P}_{\mathbf{X}}, \mathfrak{P}_{\mathbf{Y}}\right\} \equiv \Omega(\mathbf{X}, \mathbf{Y}) .
$$

Note that eq. (3.21) can be interpreted in two ways from left to right or vice-versa. Namely, given a symplectic vector field $\mathbf{X}$ we can construct its momentum-map representation $\mathfrak{P}_{\mathbf{X}}$ or, given a function $F$ on the manifold $\mathcal{M}$ we can look for the symplectic vector field $\mathbf{X}_{F}$ such that:

$$
\mathfrak{P}_{\mathbf{X}_{\mathbf{F}}}=F
$$

We can define such a construction the inverse momentum map.

A fundamental property of the momentum-map is its equivariance which amounts to the following equation:

$$
\forall \mathbf{X}, \mathbf{Y}=\text { symplectic vector fields } \quad: \quad \mathbf{Y} \mathfrak{P} \mathbf{X}=\mathfrak{P}[\mathbf{Y}, \mathbf{X}]
$$

Having recalled these concepts let us now return to the case of a canonical system endowed with Liouville integrability. According to our previous definitions this means that on the symplectic manifold $\mathcal{M}$ of dimension $2 n$ there exist $n$ independent functions $\mathcal{H}_{i}(Z)$ in involution, as stated in eq. (3.11). Let us construct the inverse momentum map of such functions, namely $n$ symplectic vector fields $\mathbf{X}_{i}$ such that:

$$
\mathfrak{P}_{\mathbf{x}_{i}}=\mathcal{H}_{i}(Z) \text {. }
$$

By construction these vector fields commute with each other and furthermore, in force of equivariance, we have:

$$
\mathbf{X}_{i} \mathcal{H}_{j}=\mathbf{X}_{i} \mathfrak{P}_{\mathbf{X}_{j}}=\mathfrak{P}_{\left[\mathbf{X}_{i}, \mathbf{x}_{j}\right]}=0 .
$$

\footnotetext{
${ }^{7}$ actually these are not true functions rather they are sections of the line-bundle defined by the transitions functions introduced in eq. (3.23).
} 
This means that the vector fields $\mathbf{X}_{i}$ are tangential to the $n$-dimensional level set surface $\Sigma_{h}$ defined by the following equations:

$$
\mathcal{H}_{i}(Z)=h_{i}
$$

where $h_{i}$ are some set of $n$ real numbers. Since the $n$ independent vector fields $\mathbf{X}_{i}$ provide a basis of sections of the tangent bundle to the level surface $T \Sigma_{h}$, it follows that the symplectic form $\Omega$ restricted to $\Sigma_{h}$ vanishes. Indeed we have:

$$
\left.\Omega\left(\mathbf{X}_{i}, \mathbf{X}_{j}\right)\right|_{\Sigma_{h}}=\left\{\mathcal{H}_{i}, \mathcal{H}_{j}\right\}=0
$$

If we use a local canonical patch $\mathcal{U} \subset \mathcal{M}_{2 n}$ of the symplectic manifold in which

$$
\Omega=\sum_{i} d P_{i} \wedge d \phi^{i}=d\left(\sum_{i} P_{i} d \phi^{i}\right),
$$

we conclude that on the level surface $\Sigma_{h}$ we have:

$$
\left.\left(d \sum_{i} P_{i} d \phi^{i}\right)\right|_{\Sigma_{h}}=0 .
$$

Being closed, the restriction to $\Sigma_{h}$ of the one-form:

$$
P=P_{i} d \phi^{i},
$$

is locally exact on the same surface. In other words on each open neighborhood $\mathcal{U} \subset \Sigma_{h}$ there exists a local function $W^{(\mathcal{U})}$ such that:

$$
P=d W^{(\mathcal{U})} .
$$

Let us now consider the definition of the level set surface:

$$
h_{i}=\mathcal{H}_{i}(\phi, P),
$$

Equations (3.35) allow us, formally, to rewrite the momenta as functions of the $\phi$, provided $\operatorname{det}\left(\partial h_{i} / \partial P_{j}\right) \neq 0$ and use the canonical coordinates $\phi^{i}$ as independent coordinates on the level surface $\Sigma_{h}$. Under these conditions, recalling eq. (3.2) we arrive at the conclusions that (locally) we have:

$$
\dot{\phi}^{i}=g^{i j}(\phi) P_{j}\left(\phi^{k}, h_{k}\right),
$$

which is the more standard Hamilton-Jacobi formulation. The $\mathcal{W}$-function $\mathcal{W}\left(\phi^{i}, h_{j}\right)$ is nothing but the Hamilton's characteristic function solution to the Hamilton-Jacobi equations $[8,58,59]$

$$
\mathcal{H}\left(\frac{\partial \mathcal{W}}{\partial \phi^{i}}, \phi^{i}\right)=\frac{1}{2} \frac{\partial \mathcal{W}}{\partial \phi^{i}} g^{i j}\left(\phi^{k}\right) \frac{\partial \mathcal{W}}{\partial \phi^{j}}=v^{2}
$$

We can say that a complete solution $\mathcal{W}\left(\phi^{i}, h_{j}\right)$ to the above equation exists in any simply connected domain of the phase space in which the Jacobian $\operatorname{det}\left(\partial h_{i} / \partial P_{j}\right) \neq 0$ is non vanishing. ${ }^{8}$

\footnotetext{
${ }^{8}$ This property is stronger than the so called "flow box theorem", see for instance [60], which implies the existence of a complete solution to the Hamilton-Jacobi equation in a neighborhood of any point in which $\left(\frac{\partial \mathcal{H}}{\partial \phi^{2}}, \frac{\partial \mathcal{H}}{\partial P_{i}}\right) \neq(0,0)$.
} 
In problems related to the study of spherically symmetric, asymptotically flat black holes, we are not interested in the complete solution $\mathcal{W}\left(\phi^{i}, h_{j}\right)$ to the Hamilton-Jacobi equation, since regularity of the four-dimensional solution implies severe restrictions on the values of the integrals $h_{i}$, as we are going to illustrate in section 5.6.

We shall apply this analysis in section 5.8 to a specific model and prove that in the domain spanned by the regular black hole solutions, the Jacobian $\operatorname{det}\left(\partial h_{i} / \partial P_{j}\right) \neq 0$ is non-vanishing. The function $\mathcal{W}$ will be explicitly derived for specific solutions.

We would now like to point out that the integration provided by the Noether charge construction

$$
P_{i}(\phi, Q)=Q_{A} \mathbb{L}^{-1}{ }_{B}^{A} V_{i}^{B},
$$

provides, generically, a non-closed momentum one-form

$$
\mathrm{d} P \neq 0
$$

see [19] for some examples of this. This means that the first order integration provided by the Noether charge is different from the first order integration provided by the HamiltonJacobi formalism and therefore the conclusions reached in [19] about the existence of the $\mathcal{W}$-function are incorrect.

In this way we have shown that Liouville integrability is completely equivalent to the existence of the so called (fake) superpotential $\mathcal{W}$. On the other hand, as it was pointed out in [48] and as it will be further discussed in a next coming paper [50], all supergravity theories where the scalar manifold is a generic, not necessarily symmetric coset manifold, possess Liouville integrability. Hence for all these cases we proved the existence of the fake superpotential. Let us also stress that we can choose one of the Hamiltonians, say $\mathcal{H}_{1}$, to coincide with the quadratic Hamiltonian $\mathcal{H}\left(Y^{A}\right)=\frac{1}{2} Y^{A} Y^{B} g_{A B}$. Its constant value $h_{1}$ will also be denoted by $v^{2}$ in the following and, for black hole solutions, it represents the extremality parameter.

\section{Lax integration algorithms}

Proving integrability is one thing; constructing the general integral of the geodesic equations is a separate issue. In this section, relying on earlier results [37, 40, 41, 48, 54-56], we show that this general integral can be constructed. All integration algorithms so far developed, focus on giving a solution for the Lax operator $L(\tau)$. As explained under (2.20), this is in principle sufficient, as it allows to obtain the solutions for the scalar fields after solving a second system of differential equations that can be solved explicitly. For practical reasons, it is however desirable to circumvent this second integration step, by giving an integration formula for the coset representative. In this section, we will propose and prove such an integration formula. In section 4.1, we will review how the integration for the Lax operator is performed. Relying on that result, the formula for the coset representative will be discussed in section 4.2 . 


\subsection{Integration formula for the Lax operator}

Let us repeat here the results of [40]. We will assume that we work in a matrix representation of $\mathfrak{g}$ consisting of $N \times N$-matrices. The authors of [40] showed that the solution for the matrix elements $L_{p q}(\tau)$ of the Lax operator can be given in closed form, according to a very specific integration formula that depends on an initial value $L_{0}=L(\tau=0)$ for the Lax operator. In fact, several equivalent versions of these formulas were given.

The integration formula for $L(\tau)$ that we start from is given by

$$
L(\tau)=\mathcal{Q}(\mathcal{C}) L_{0}(\mathcal{Q}(\mathcal{C}))^{-1},
$$

where the matrix $\mathcal{Q}(\mathcal{C})$ has the following elements

$$
\mathcal{Q}_{i j}(\mathcal{C}) \equiv \frac{1}{\sqrt{\mathfrak{D}_{i}(\mathcal{C}) \mathfrak{D}_{i-1}(\mathcal{C})}} \operatorname{Det}\left(\begin{array}{cccc}
\mathcal{C}_{1,1}(\tau) & \ldots & \mathcal{C}_{1, i-1}(\tau) & \left(\mathcal{C}^{\frac{1}{2}}(\tau)\right)_{1, j} \\
\vdots & \vdots & \vdots & \vdots \\
\mathcal{C}_{i, 1}(\tau) & \ldots & \mathcal{C}_{i, i-1}(\tau) & \left(\mathcal{C}^{\frac{1}{2}}(\tau)\right)_{i, j}
\end{array}\right)
$$

and we have defined

$$
\begin{gathered}
\mathcal{C}(\tau):=\mathrm{e}^{-2 \tau L_{0}} \\
\mathfrak{D}_{i}(\mathcal{C}):=\operatorname{Det}\left(\begin{array}{ccc}
\mathcal{C}_{1,1}(\tau) & \ldots & \mathcal{C}_{1, i}(\tau) \\
\vdots & \vdots & \vdots \\
\mathcal{C}_{i, 1}(\tau) & \ldots & \mathcal{C}_{i, i}(\tau)
\end{array}\right), \quad \mathfrak{D}_{0}(\tau):=1
\end{gathered}
$$

The formula (4.1) can be proven by showing that it obeys the Lax equation. In order to do this, we start by using $\mathcal{Q}(\mathcal{C})$ to define the following triangular matrices

$$
\begin{aligned}
\mathcal{X}_{>}(\mathcal{C}) & :=\mathcal{Q}(\mathcal{C}) \mathcal{C}^{\frac{1}{2}}(\tau), \\
\left(\mathcal{X}_{<}(\mathcal{C})\right)^{-1} & :=\mathcal{Q}(\mathcal{C})\left(\mathcal{C}^{\frac{1}{2}}(\tau)\right)^{-1}
\end{aligned}
$$

One can then show that $\mathcal{X}_{>}(\mathcal{C})$ (as well as its inverse) is upper-triangular. Similarly, it can be seen that $\mathcal{X}_{<}(\mathcal{C})$ (as well as its inverse) is lower-triangular. Another useful property of these matrices is that their diagonal elements are equal

$$
\left(\mathcal{X}_{>}(\mathcal{C})\right)_{i i}=\left(\mathcal{X}_{<}(\mathcal{C})\right)_{i i}
$$

The proof of (4.1) then starts by writing the matrix $\mathrm{e}^{-\tau L_{0}}$ in terms of $\mathcal{X}_{>}(\mathcal{C})$ and $\mathcal{X}_{<}(\mathcal{C})$

$$
\begin{aligned}
\mathrm{e}^{-\tau L_{0}} & =(\mathcal{Q}(\mathcal{C}))^{-1} \mathcal{X}_{>}(\mathcal{C}), \\
\mathrm{e}^{-\tau L_{0}} & =\mathcal{X}_{<}(\mathcal{C}) \mathcal{Q}(\mathcal{C}) .
\end{aligned}
$$

By deriving (4.8) and (4.9) with respect to $\tau$, one can obtain

$$
\begin{aligned}
& \mathcal{Q}(\mathcal{C}) \frac{d}{d \tau}(\mathcal{Q}(\mathcal{C}))^{-1}=-L(\tau)-\left(\frac{d}{d \tau} \mathcal{X}_{>}(\mathcal{C})\right)\left(\mathcal{X}_{>}(\mathcal{C})\right)^{-1} \\
& \mathcal{Q}(\mathcal{C}) \frac{d}{d \tau}(\mathcal{Q}(\mathcal{C}))^{-1}=+L(\tau)+\left(\mathcal{X}_{<}(\mathcal{C})\right)^{-1}\left(\frac{d}{d \tau} \mathcal{X}_{<}(\mathcal{C})\right)
\end{aligned}
$$


Using these equations, the triangularity properties of $\mathcal{X}_{>}(\mathcal{C})$ and $\mathcal{X}_{<}(\mathcal{C})$ and $(4.7)$, one can see that

$$
\mathcal{Q}(\mathcal{C}) \frac{d}{d \tau}(\mathcal{Q}(\mathcal{C}))^{-1}=L_{>}(\tau)-L_{<}(\tau)=W(\tau)
$$

By deriving (4.1) with respect to $\tau$, one finds

$$
\frac{d}{d \tau} L(\tau)+\left[\mathcal{Q}(\mathcal{C}) \frac{d}{d \tau}(\mathcal{Q}(\mathcal{C}))^{-1}, L(\tau)\right]=0
$$

so that with (4.12) we find that $L(\tau)$ indeed obeys the Lax equation.

The formula (4.1) gives an integration formula for the Lax operator $L(\tau)$. As explained above, in order to obtain the explicit expressions for the scalar fields, one still needs to perform an extra integration. We can actually do better and give an integration formula for the coset representative $\mathbb{L}(\tau)$. As the latter is solely expressed in terms of the scalar fields and no longer in terms of their derivatives, one can use it to easily extract the scalar field solutions.

\subsection{An integration formula for the coset representative}

We now derive an integration formula for the inverse coset representative. Throughout this derivation, we will assume that we are working on the coset space $\frac{\mathrm{S} \ell(p+q)}{\mathrm{SO}(p, q)}$. This is not really a restriction, as one can always find values for $p$ and $q$ and a representation of $G$, such that the coset space $G / H^{*}$ can be embedded in $\frac{S L(p+q)}{S O(p, q)}$

$$
\frac{G}{H^{*}} \hookrightarrow \frac{S L(p+q)}{S O(p, q)}
$$

As according to this embedding the Lax connection is an element of the algebra of $\mathrm{SO}(p, q)$, we have

$$
W(\tau) \eta(p, q)=-\eta(p, q) W^{T}(\tau)
$$

where

$$
\eta(p, q):=\left(\begin{array}{cc}
+\mathbb{1}_{p} & 0 \\
0 & -\mathbb{1}_{q}
\end{array}\right), \quad p+q=N .
$$

Similarly, the Lax operator $L(\tau)$ obeys

$$
L(\tau) \eta(p, q)=\eta(p, q) L^{T}(\tau) .
$$

We can now use the fact that $L$ and $W$ can be represented according to the formulae

$$
\begin{aligned}
L(\tau) & =L_{>}(\tau)+L_{<}(\tau)-\operatorname{diag}(L(\tau)) \\
W(\tau) & =L_{>}(\tau)-L_{<}(\tau)
\end{aligned}
$$

and substituting eqs. (4.18)-(4.19) into eq. (2.15) we obtain the following relation

$$
\mathbb{L}(\tau)^{-1} \frac{d}{d \tau}(\mathbb{L}(\tau))=2 L_{>}(\tau)-\operatorname{diag}(L(\tau)) .
$$


On the other hand, let us note that by summing (subtracting) eqs. (4.10) and (from) (4.11) one obtains

$$
\begin{aligned}
\mathcal{Q}(\mathcal{C}) \frac{d}{d \tau} \mathcal{Q}^{-1}(\mathcal{C}) & =\frac{1}{2}\left[\left(\mathcal{X}_{<}(\mathcal{C})\right)^{-1}\left(\frac{d}{d \tau} \mathcal{X}_{<}(\mathcal{C})\right)-\left(\frac{d}{d \tau} \mathcal{X}_{>}(\mathcal{C})\right)\left(\mathcal{X}_{>}(\mathcal{C})\right)^{-1}\right] \\
L(\tau) & =-\frac{1}{2}\left[\left(\mathcal{X}_{<}(\mathcal{C})\right)^{-1}\left(\frac{d}{d \tau} \mathcal{X}_{<}(\mathcal{C})\right)+\left(\frac{d}{d \tau} \mathcal{X}_{>}(\mathcal{C})\right)\left(\mathcal{X}_{>}(\mathcal{C})\right)^{-1}\right]
\end{aligned}
$$

Comparing these equations with (4.12) allows us to express the right hand side of (4.20) as

$$
2 L_{>}(\tau)-\operatorname{diag}(L(\tau))=\mathcal{X}_{>}(\mathcal{C}) \frac{d}{d \tau}\left(\mathcal{X}_{>}(\mathcal{C})\right)^{-1}
$$

A simple comparison of equations (4.23) and (4.20) shows that

$$
\begin{aligned}
\left(\mathbb{L}(\tau)^{-1}\right)_{i j} & =\left(\mathcal{X}>(\mathcal{C}) \mathbb{L}(0)^{-1}\right)_{i j} \\
& \equiv \frac{1}{\sqrt{\mathfrak{D}_{i}(\mathcal{C}) \mathfrak{D}_{i-1}(\mathcal{C})}} \operatorname{Det}\left(\begin{array}{cccc}
\mathcal{C}_{1,1}(\tau) & \ldots & \mathcal{C}_{1, i-1}(\tau) & \left(\mathcal{C}(\tau) \mathbb{L}(0)^{-1}\right)_{1, j} \\
\vdots & \vdots & \vdots & \vdots \\
\mathcal{C}_{i, 1}(\tau) & \ldots & \mathcal{C}_{i, i-1}(\tau) & \left(\mathcal{C}(\tau) \mathbb{L}(0)^{-1}\right)_{i, j}
\end{array}\right)
\end{aligned}
$$

where we have used that from its definition

$$
\left(\mathcal{X}_{>}(\mathcal{C})\right)_{i j} \equiv \frac{1}{\sqrt{\mathfrak{D}_{i}(\mathcal{C}) \mathfrak{D}_{i-1}(\mathcal{C})}} \operatorname{Det}\left(\begin{array}{cccc}
\mathcal{C}_{1,1}(\tau) & \ldots & \mathcal{C}_{1, i-1}(\tau) & \mathcal{C}_{1, j}(\tau) \\
\vdots & \vdots & \vdots & \vdots \\
\mathcal{C}_{i, 1}(\tau) & \ldots & \mathcal{C}_{i, i-1}(\tau) & \mathcal{C}_{i, j}(\tau)
\end{array}\right)
$$

Note that we have introduced a constant matrix $\mathbb{L}(0) \in \frac{\mathrm{S} \ell(p+q)}{\mathrm{SO}(p, q)}$ in the solvable parameterization of the coset. This matrix can be seen as a matrix of integration constants. The formula (4.24) describes an explicit general solution for the coset representative $\mathbb{L}(\tau) \in \frac{\mathrm{S} \ell(p+q)}{\mathrm{SO}(p, q)}$. This solution is parameterized by the initial data $L_{0}$ and $\mathbb{L}(0)$, which gives precisely the correct number of integration constants associated to the geodesic equations.

\section{Applications to black holes}

\subsection{Black holes as geodesics}

As explained in the introduction, stationary black holes in $d+1$ dimensions can be reduced over time to Euclidean solutions in $d$ dimensions. From now on, we assume that $d=3$, but the extension to any dimension is straightforward, see e.g. [25].

Consider a four-dimensional supergravity describing scalar fields $\phi^{r}$, and $n_{V}$ vector fields $B^{I}, I=1, \ldots, n_{V}$. We suppose the scalar manifold to be homogeneous-symmetric of the form $G_{4} / H_{4}, G_{4}$ being the isometry group of the manifold and $H_{4}$ its maximal compact 
subgroup. The action in four dimensions is given by ${ }^{9}$

$$
S_{4}=\int\left(\frac{1}{2} \star R_{4}-\frac{1}{2} G_{r s} \star \mathrm{d} \phi^{r} \wedge \mathrm{d} \phi^{s}-\frac{1}{2} \mu_{I J} \star G^{I} \wedge G^{J}+\frac{1}{2} \nu_{I J} G^{I} \wedge G^{J}\right),
$$

where $G^{I}=\mathrm{d} B^{I}$, and $G_{r s}, \mu_{I J}, \nu_{I J}$ are symmetric matrices that depend on the scalars $\phi$; in particular, $G$ and $\mu$ are required to be positive definite. The group $G_{4}$ represents the on-shell global symmetry group of the theory, once its non-linear action on the scalars $\phi^{r}$ is supplemented by a linear (symplectic) electric-magnetic duality transformation on the vector field strengths and their magnetic duals.

Let us now restrict ourselves to stationary solutions having an $\mathrm{SO}(3)$ spatial isometry, which comprise static black holes though we may allow also for a NUT charge. We then "reduce" the action over the time-like direction using the following ansatz

$$
\begin{aligned}
\mathrm{d} s_{4}^{2} & =-\mathrm{e}^{2 U}\left(\mathrm{~d} t+A_{K K}\right)^{2}+\mathrm{e}^{-2 U} \mathrm{~d} s_{3}^{2}, \\
B^{I} & =\tilde{B}^{I}+Z^{I}\left(\mathrm{~d} t+A_{K K}\right),
\end{aligned}
$$

where $\tilde{B}^{I}$ and $A_{K K}$ are vectors in $d=3+0$ and $U$ and $Z^{I}$ are scalar fields. Since time is not periodic this is not a compactification over time, but the difference between a dimensional reduction over a compact or a non-compact direction only enters the KK sector, which is assumed truncated here.

When $\mathrm{d} A_{K K} \neq 0$ the $3 d$ vector $A_{K K}$ can not be removed using a coordinate transformation and this off-diagonal term in the metric then corresponds to Taub-NUT charge.

After dimensional reduction we dualize the vector fields $\tilde{B}^{I}$ and $A_{K K}$ to scalars $Z_{I}, a$, see appendix A for the details of this derivation, so to end up with an Euclidean threedimensional theory consisting of a sigma model describing $n$ scalar fields $\phi^{i}$ coupled to gravity. The fields $\phi^{i}$ consist of the four-dimensional scalars $\phi^{r}$, two scalars $U, a$ coming from the four-dimensional metric, and $2 n_{V}$ scalar fields $Z^{I}, Z_{I}$ originating from the fourdimensional vector fields and which will be collected in a $2 n_{V}$-symplectic vector $\mathbf{Z} \equiv$ $\left(Z^{M}\right)=\left(Z^{I}, Z_{I}\right)$. The target space of the sigma model is a homogeneous-symmetric pseudo-Riemannian space of the form $G / H^{*}, G$ being its isometry group and $H^{*}$ a noncompact maximal subgroup of $G$. $G$ is the global symmetry group of the three-dimensional theory and contains a subgroup of the form $\operatorname{SL}(2, \mathbb{R})_{E} \times G_{4}$, where $\operatorname{SL}(2, \mathbb{R})_{E}$ is the Ehlers group acting transitively on the scalars $U, a$.

The ansatz for the solutions in three dimensions is such that the equations of motion for the scalar fields $\phi^{i}$, decouple from the equations for the metric degrees of freedom. To make this clear we use the following ansatz

$$
\mathrm{d} s_{3}^{2}=g_{(3) m n} \mathrm{~d} x^{m} \mathrm{~d} x^{n}=\exp [4 A(\tau)] \mathrm{d} \tau^{2}+\exp [2 A(\tau)] \mathrm{d} \Omega_{2}^{2}, \quad \phi^{i}=\phi^{i}(\tau),
$$

\footnotetext{
${ }^{9}$ We use the more compact form notation here. A $p$-form $A_{p}$ is written in components as $A_{p}=$ $\frac{1}{p !} A_{\mu_{1} \ldots \mu_{p}} \mathrm{~d} x^{\mu_{1}} \wedge \ldots \wedge \mathrm{d} x^{\mu_{p}}$. The hodge star in $d$ dimensions is defined via, $\star \mathrm{d} x^{\mu_{1}} \wedge \ldots \wedge \mathrm{d} x^{\mu_{p}}=$ $\frac{1}{(p-d) !} \epsilon_{\nu_{1} \ldots, \nu_{d-p}}^{\mu_{1} \ldots \mu_{p}} \mathrm{~d} x^{\nu_{1}} \wedge \ldots \wedge \mathrm{d} x^{\nu_{d-p}}$, where $\epsilon$ is the totally anti symmetric tensor (not symbol). This implies that $\star \star A_{p}=(-1)^{p(d-p)+t} A_{p}$, where $t$ is the number of timelike dimensions in the $d$-dimensional space. We furthermore have the relations $\star A_{p} \wedge B_{p}=\star B_{p} \wedge A_{p}=\frac{1}{p !} A_{\mu_{1} \ldots \mu_{p}} B^{\mu_{1} \ldots \mu_{p}} \star 1$. The exterior derivative acts as $\mathrm{d} A_{p}=\frac{1}{p !} \partial_{\mu_{0}} A_{\mu_{1} \ldots \mu_{p}} \mathrm{~d} x^{\mu_{0}} \wedge \mathrm{d} x^{\mu_{1}} \wedge \ldots \wedge \mathrm{d} x^{\mu_{p}}$.
} 
where $\left(x^{m}\right)=(\tau, \theta, \varphi)$ are the space coordinates and $\mathrm{d} \Omega_{2}^{2}$ is the metric on the unit 2-sphere. The radial variable $\tau$ is chosen so that $\sqrt{\left|g_{(3)}\right|} g_{(3)}^{\tau \tau}$ be $\tau$-independent. Consistently with our symmetry requirements all the scalar fields will depend on $\tau$ only: $\phi^{i}=\phi^{i}(\tau)$. The scalar field equations of motion can then be derived from the geodesic action (we refer the reader to appendix A for a derivation of this action)

$$
\mathcal{L}=\frac{1}{2} g_{i j}(\phi) \dot{\phi}^{i} \dot{\phi}^{j}=\dot{U}^{2}+\frac{1}{2} G_{r s} \dot{\phi}^{r} \dot{\phi}^{s}+\frac{1}{4} \mathrm{e}^{-4 U}\left(\dot{a}+\mathbf{Z}^{T} \mathbb{C} \dot{\mathbf{Z}}\right)^{2}-\frac{1}{2} \mathrm{e}^{-2 U} \dot{\mathbf{Z}}^{T} \mathcal{M}_{4} \dot{\mathbf{Z}},
$$

where the negative definite, symmetric, symplectic matrix $\mathcal{M}_{4}$ is defined in appendix A. The target space metric is indefinite, the negative-signature directions corresponding to $\mathrm{d} Z^{M}$.

One thus finds that $\tau$ corresponds to the affine parameter along the geodesic and it is a re-parametrization of the usual radial coordinate used for spherically symmetric solutions. A geodesic that is parametrized by an affine parameter $\tau$ moves at constant speed, denoted $v^{2}$

$$
\frac{1}{2} g_{i j}(\phi) \dot{\phi}^{i} \dot{\phi}^{j} \equiv v^{2}
$$

Note that $v^{2}$ can be zero, positive or negative, due to the indefinite signature of the target space. Note that the effective Lagrangian (5.4) describes an autonomous system in which the radial variable $\tau$ plays the role of time and eq. (5.5) represents the Hamiltonian constraint. We do not consider timelike geodesics $\left(v^{2}<0\right)$ as they can be shown to correspond to over-extremal (singular) black hole solutions. Not every geodesic with $v^{2} \geq 0$ corresponds to a regular black hole solution as we explain in some detail below. For black hole solutions $v$ is the extremality parameter and is expressed as the product of the black hole temperature $T$ and entropy $S$ [4]

$$
v=2 S T \text {. }
$$

Hence, extremal solutions, for which $v=0$, are described by lightlike geodesics and nonextremal solutions by spacelike geodesics.

The solution for the metric is independent of the details of the sigma model and is determined by the geodesic velocity only. ${ }^{10}$ It can be found to be

$$
\begin{aligned}
v^{2}>0: & \exp [2 A]=\frac{v^{2}}{\sinh ^{2}(v \tau)}, \\
v^{2}=0: & \exp [2 A]=\frac{1}{\tau^{2}} .
\end{aligned}
$$

The non-trivial part in finding the three-dimensional solutions using the ansatz (5.3) thus consists of solving the geodesic equations for the scalar fields $\phi^{i}$. One of the scalars in three dimensions is the Kaluza-Klein dilaton $U$ that comes from the dimensional reduction over time. The three-dimensional solutions are uplifted to four dimensions using equations (5.2).

\footnotetext{
${ }^{10}$ This expression is sensitive to numerical factors that depend on the normalisation of the Einstein-Hilbert term with respect to the normalisation of the scalar kinetic term.
} 


\subsection{The structure of $\mathfrak{g}$}

For homogenous sigma models that appear upon time-like dimensional reduction from four dimensions [23, 25], the adjoint representation of the isometry group $G$ branches with respect to its $\mathrm{SL}(2, \mathbb{R})_{E} \times G_{4}$ subgroup, upon dualization of all the vector fields to scalar fields as follows

$$
\operatorname{Adj}[G] \rightarrow\left(\operatorname{Adj}\left[\operatorname{SL}(2, \mathbb{R})_{E}\right], \mathbf{1}\right) \oplus\left(\mathbf{1}, \mathbf{A d j}\left[G_{4}\right]\right) \oplus(\mathbf{2}, \mathbf{R}),
$$

where $\mathbf{R}$ is the symplectic representation in which the electric and magnetic charges of the four-dimensional theory transform under the duality action of $G_{4}$.

The solvable algebra Solv of (2.5) has a specific structure [25, 42]

$$
\text { Solv }=\operatorname{Solv}_{2} \oplus \operatorname{Solv}_{4} \oplus \operatorname{Span}\left(T_{M}\right),
$$

where $\operatorname{Solv}_{2}=\operatorname{Span}\left(H_{0}, E_{\beta_{0}}\right)$ generates a submanifold $\mathrm{S} \ell(2, \mathbb{R}) / \mathrm{SO}(2), \beta_{0}$ being the highest root of $\mathfrak{g}$, while $S_{\text {olv }}$ generates the scalar manifold $G_{4} / H_{4}$ of the four-dimensional parent theory. The nilpotent generators $T_{M}, M=1, \ldots, 2 n_{V}$ correspond to the positive (restricted) roots $\gamma_{M}$ defined by the property $\gamma_{M}\left(H_{0}\right)=1$. The space $\operatorname{Span}\left(T_{M}\right)$ transforms in the representation $\mathbf{R}_{+1}$ with respect to the subgroup $G_{4} \times \mathrm{O}(1,1)$ of $G$, the $\mathrm{O}(1,1)$ factor being generated by $H_{0}$. The algebraic structure of $S o l v$ is described as follows

$$
\begin{aligned}
{\left[\text { Solv }_{2}, \text { Solv }_{4}\right] } & =0, & & {\left[H_{0}, E_{\beta_{0}}\right]=2 E_{\beta_{0}}, } \\
{\left[H_{0}, T_{M}\right] } & =T_{M}, & {\left[T_{M}, T_{N}\right] } & =\mathbb{C}_{M N} E_{\beta_{0}},
\end{aligned}
$$

where $\mathbb{C}_{M N}$ is the symplectic invariant matrix.

At this point we have partially fixed normalisations through equation (5.11). If we furthermore fix the normalisation of $\mathbb{C}$ such that it has only \pm 1 as entries then there is one scaling freedom left; we can rescale $E_{\beta_{0}}$ if we accordingly rescale the $T_{M}$ generators.

Let us now describe the structure of $\mathfrak{g}$ and of the subspaces $\mathfrak{K}, \mathfrak{H}^{*}$ in the Cartan decomposition (2.1). The matrix $\eta$ defining the decomposition through the involution $\theta$ (2.2) has the following intrinsic expression in terms of the Cartan generator $H_{0}$ : $\theta=$ $(-1)^{H_{0}}$. It is easy to verify from (5.11) that

$$
\eta T_{M} \eta=-T_{M}, \quad \eta T_{r} \eta=T_{r}, \quad \eta E_{\beta_{0}} \eta=E_{\beta_{0}} .
$$

For notational convenience we define the basis of generators $\left\{T_{A}\right\}$ of $S$ olv as follows: $\left\{T_{A}\right\}=$ $\left\{T_{0}, T_{r}, T_{M}, T_{\bullet}\right\}$ where $T_{0}=\frac{H_{0}}{2}, T_{\bullet}=E_{\beta_{0}}$ and $\left\{T_{r}\right\}$ is a basis of $S_{0 l v_{4}}$. From the above properties it follows that the generators $K_{A}$ of the space $\mathfrak{K}$, defined in terms of $T_{A}$ by eq. (2.6) have the following form

$$
\begin{aligned}
\left\{K_{A}\right\} & =\left\{K_{0}, K_{r}, K_{M}, K_{\bullet}\right\}, \quad K_{0}=T_{0}, K_{r}=\frac{1}{2}\left(T_{r}+T_{r}^{T}\right), K_{\bullet}=\frac{1}{2}\left(T_{\bullet}+T_{\bullet}^{T}\right), \\
K_{M} & =\frac{1}{2}\left(T_{M}-T_{M}^{T}\right) .
\end{aligned}
$$

The non-compact generators $K_{r}$ generate the space $\mathfrak{K}_{4}$ in the Cartan decomposition of the algebra $\mathfrak{g}_{4}$ of $G_{4}$ with respect to its maximal compact subalgebra $\mathfrak{H}_{4}: \mathfrak{g}_{4}=\mathfrak{H}_{4} \oplus \mathfrak{K}_{4}$. The 
non-compact generators $\left\{K_{0}, K_{\bullet}\right\}$ generate the space $\mathfrak{K}_{2}$ of the Cartan decomposition of the Ehlers algebra $\mathfrak{s l}(2, \mathbb{R})_{E}$ with respect to its maximal compact subalgebra $\mathfrak{u}(1)_{E}: \mathfrak{s l}(2, \mathbb{R})_{E}=$ $\mathfrak{u}(1)_{E} \oplus \mathfrak{K}_{2}$. Finally the compact generators $K_{M}$ span a $2 n_{V}$-dimensional space $\mathfrak{K}_{\mathbf{R}_{c}}$.

The metric $g_{A B}$ at the origin of the manifold is proportional to the restriction to $\mathfrak{K}$ of the Cartan-Killing metric on $\mathfrak{g}$, and thus it has $2 n_{V}$ negative signature directions corresponding to the compact generators $K_{M}$. The space $\mathfrak{K}$ transforms, according to equation (2.3) in a linear representation with respect to the adjoint action of $H^{*}$. In particular we can consider the adjoint action on $\mathfrak{K}$ of the maximal compact subgroup $H_{c}$ of $H^{*}$. This group has the general form $H_{c}=\mathrm{U}(1)_{E} \times H_{4}, H_{4}$ being the maximal compact subgroup of $G_{4}$. The compact symmetry group $H_{4}$, which acts linearly on the central and matter charges in four dimensions, is enhanced in the three-dimensional theory by the $\mathrm{U}(1)_{E}$ subgroup of the Ehlers group. With respect to the adjoint action of $H_{c}$, the subspace $\mathfrak{K}_{\mathbf{R}_{c}}$ spanned by $\left\{K_{M}\right\}$, transforms in a representation $\mathbf{R}_{c}$. This is the representation in which the central and matter charges in four dimensions transform with respect to $H_{c}$. For instance, in the maximally supersymmetric theory, $G_{4}=\mathrm{E}_{7(7)}, H_{4}=\mathrm{SU}(8), G=\mathrm{E}_{8(8)}$ and $H^{*}=\mathrm{SO}^{*}(16)$. The group $H_{c}$ is $\mathrm{U}(8)=\mathrm{U}(1)_{E} \times \mathrm{SU}(8)$ and $\mathbf{R}_{c}=\mathbf{2 8}_{+1}+\overline{\mathbf{2 8}}_{-1}$, the subscripts being the $\mathrm{U}(1)_{E}$ gradings. In this case we can choose complex basis elements $K_{M}$, labeled by antisymmetric couples $[a b], a, b=1, \ldots, 8:\left(K_{M}\right)=\left(K_{a b}, K^{a b}\right)$.

The Lax operator $L$ is defined to be an element of $\mathfrak{K}$. Its components along the generators $K_{M}$ are the central and matter charges of the four-dimensional theory, which depend on the quantized charges and the scalar fields. For instance in the maximal theory

$$
\left.L\right|_{\mathfrak{R}_{\mathbf{R}_{c}}}=\sqrt{2}\left(\bar{Z}^{a b} K_{a b}+Z_{a b} K^{a b}\right),
$$

$Z_{a b}$ being the complex central charges of the four-dimensional $\mathcal{N}=8$ parent theory. It is known that a complex $8 \times 8$ matrix can be skew-diagonalized by means of a $\mathrm{U}(8)$ transformation. The 4 real skew-eigenvalues $\rho_{k}$ of the central charge matrix $Z_{a b}$ define the normal form of the four-dimensional central charges with respect to the action of $H_{c}$. From a three-dimensional point of view, this procedure amounts to rotating $\left.L\right|_{\mathfrak{K}_{\mathbf{R}_{c}}}$, by means of the adjoint action of $H_{c}$, into a 4-dimensional abelian subalgebra $\mathfrak{K}_{N}=\left\{K_{k}\right\}$ of $\mathfrak{K}_{\mathbf{R}_{c}}$

$$
\exists h \in H_{c}:\left.h^{-1} L\right|_{\mathfrak{K}_{\mathbf{R}_{c}}} h=\sqrt{2} \sum_{k=1}^{4} \rho_{k} K_{k} .
$$

We can generalize the above discussion to a generic theory and define the normal form of $\mathbf{R}_{c}$ with respect to $H_{c}$ as the minimal subspace (normal subspace) of its basis into which any of its elements can be rotated by means of an $H_{c}$-transformation. Let us denote by $p$ the dimension of this subspace. Thus $p$ is the minimal number of charges into which the most general set of central and matter charges, in $\mathbf{R}_{c}$, can be reduced by means of an $H_{c}$-transformation. In the maximal theory we have seen that $p=4$. This implies that the most general black hole solution modulo the action of the three-dimensional global symmetry group $G$ (the generating or seed solution) is a p-charge solution. Taking $\mathfrak{K}_{\mathbf{R}_{c}}$ as the basis of the $\mathbf{R}_{c}$-representation, it was shown in [25] that its normal subspace $\mathfrak{K}_{N}=\left\{K_{\ell}\right\}$ is spanned by $p$-commuting generators $K_{\ell}, \ell=1, \ldots, p$, where $p$ is the rank 
of the symmetric Riemannian coset $H^{*} / H_{c}: p=\operatorname{rank}\left(\frac{H^{*}}{H_{c}}\right)$. In the maximal theory, $p=$ $\operatorname{rank}\left(\frac{H^{*}}{H_{c}}\right)=\operatorname{rank}\left(\frac{\mathrm{SO}^{*}(16)}{\mathrm{U}(8)}\right)=4$. Similarly we can define a space $\mathfrak{H}_{\mathbf{R}_{c}}$ of non-compact generators from the Cartan decomposition of $\mathfrak{H}^{*}$ with respect to the lie algebra $\mathfrak{H}_{c}$ of $H_{c}$ : $\mathfrak{H}^{*}=\mathfrak{H}_{c} \oplus \mathfrak{H}_{\mathbf{R}_{c}}$. Just as $\mathfrak{K}_{\mathbf{R}_{c}}$, also $\mathfrak{H}_{\mathbf{R}_{c}}$ defines, with respect to the adjoint action of $H_{c}$, the basis of a representation $\mathbf{R}_{c}$. We can then define a normal subspace $\mathfrak{H}_{N}=\left\{J_{\ell}\right\}$, $\ell=1, \ldots, p$, of $\mathfrak{H}_{\mathbf{R}_{c}}$ with respect to the action of $H_{c}$. The non-compact generators $J_{\ell}$ of the normal subspace, can be chosen together with the compact counterparts $K_{\ell}$ in $\mathfrak{K}_{\mathbf{R}_{c}}$ so that, $K_{\ell}, J_{\ell}$ and $H_{\ell} \equiv\left[K_{\ell}, J_{\ell}\right]$ generate $p$ commuting $S L(2, \mathbb{R})$ subgroups of $G$. These groups define a subspace of $G / H^{*}$ of the form

$$
\left(\frac{\mathrm{SL}(2, \mathbb{R})}{\mathrm{SO}(1,1)}\right)^{p} \subset \frac{G}{H^{*}}
$$

which consists of the $p$-fold product of $d S_{2}$ spaces. This space and its generating isometries plays a role in the construction of the seed solutions, relative to the action of $G$, of regular black holes [25].

\subsection{The solvable parametrization and the Noether charges}

What we would like to stress from the above discussion is that the $p$ commuting compact generators $K_{\ell}$ in the coset generate a $p$-torus $T^{p}$ inside $G / H^{*}$. In the case of a $d S_{2}$ space, for instance, $p=1$ and the torus is the non-trivial 1-cycle $S_{1}$ of the corresponding hyperboloid. A generic element of $T^{p}$ can not in general be brought to the form of a solvable group element of exp Solv times an element of $H^{*}$. This can be easily seen in the case of the space $d S_{2}$ : The circle $S_{1}$ at fixed global time interpolates between two solvable patches, each describing a copy of Einstein's static universe (see [41] for a detailed discussion of this issue). In general each 1-cycle of $T^{p}$ interpolates between different solvable patches in $G / H^{*}$. If one solvable patch is described by a coset representative $\mathbb{L}(\phi) \in \exp (\operatorname{Solv})$, the manifold being homogeneous, we can find a compact element $\mathcal{D}$ of $G / H^{*}$ which maps the origin of this patch into a point in any other solvable patch, this being described by a coset representative of the form $\mathcal{D} \cdot \mathbb{L}(\phi) \in \mathcal{D} \cdot \exp (S o l v)$. Thus homogeneity of $G / H^{*}$ implies that any two solvable patches are isometric images of each other. As pointed out in [29], the subset of points covered by the solvable patches is dense in $G / H^{*}$ and the points which are at the boundary of the various patches, and thus are not described by the solvable parametrization, are those in which $\mathrm{e}^{-U}=0$, where $U$ is the scalar entering the metric in the ansatz (5.2), and that parametrizes the Cartan generator $T_{0}$ of $S o l v$, see below. Solutions crossing the boundary between two solvable patches are characterized by the property that $\mathrm{e}^{-U}$ vanishes at some finite $\tau$, and are therefore singular. It was shown in [20] for geodesics in $d S_{2}$, that only the space-like ones cross the boundary of the solvable patch, while the other geodesics unfold within the same solvable patch. Physical fields belong to a single solvable patch (physical patch) and, as long as we are interested in regular solutions only, we can restrict ourselves to the physical patch only, since in doing so, we are not loosing any physics. We can then define the scalar manifold as a solvable symmetric pseudo-Riemannian manifold obtained by identifying all solvable patches of 
$G / H^{*}:$

$$
\mathcal{M}_{\text {scal }} \equiv D \backslash G / H^{*},
$$

where $D$ is the discrete subgroup in $G / H^{*}$ whose elements map solvable patches into one another.

Let us now define our solvable parametrization. It is convenient to define the coset representative $\mathbb{L}$ in the solvable gauge as follows

$$
\mathbb{L}\left(\phi^{i}\right)=\mathrm{e}^{-a E_{\beta_{0}}} \mathrm{e}^{\sqrt{2} Z^{M} T_{M}} \mathbb{L}_{4}\left(\phi^{r}\right) \mathrm{e}^{U H_{0}},
$$

where $\mathbb{L}_{4}$ is the coset representative of $G_{4} / H_{4}$. Using the coset representative (5.18) one can calculate the sigma model metric, the Lax operator $L$ and Lax connection $W$ explicitly.

The structure constants of Solv, in the basis $\left\{T_{0}, T_{r}, T_{M}, T_{\bullet}\right\}$, have the following nonvanishing components

$$
\begin{gathered}
{\left[T_{A}, T_{B}\right]=f_{A B}{ }^{C} T_{C} ;} \\
f_{0 M}{ }^{N}=\frac{1}{2} \delta_{M}^{N} ; f_{0} \bullet^{\bullet}=1 ; f_{r s}{ }^{t} ; f_{r M}{ }^{N}=-\left(T_{r}\right)_{M}{ }^{N} ; f_{M N} \bullet=\mathbb{C}_{M N} .
\end{gathered}
$$

We find, after some calculations,

$$
\begin{aligned}
& \mathbb{L}_{0}^{0}=1 \\
& \mathbb{L}_{0}{ }^{M}=\frac{\mathrm{e}^{-U}}{\sqrt{2}} Z^{N} \mathbb{L}_{4 N}{ }^{M} ; \quad \quad \mathbb{L}_{0} \cdot=-\mathrm{e}^{-2 U} a \\
& \mathbb{L}_{r}{ }^{\hat{s}}=\mathbb{L}_{4 r}{ }^{\hat{s}}, \\
& \mathbb{L}_{r}{ }^{M}=-\sqrt{2} \mathrm{e}^{-U}\left(\mathbf{Z}^{T} T_{r} \mathbb{L}_{4}\right)^{M} ; \quad \mathbb{L}_{r}^{\bullet}=-\mathrm{e}^{-2 U} T_{r M N} Z^{M} Z^{N} ; \\
& \mathbb{L}_{M}{ }^{N}=\mathrm{e}^{-U} \mathbb{L}_{4 M}{ }^{N}, \quad \quad \mathbb{L}_{M}^{\bullet}=\sqrt{2} \mathrm{e}^{-2 U} \mathbb{C}_{M N} Z^{N} ; \quad \mathbb{L}_{\bullet}^{\bullet}=\mathrm{e}^{-2 U} .
\end{aligned}
$$

In (5.20) we have used the $d=4$ coset representative $\mathbb{L}_{4}$ and the $\operatorname{Solv}_{4}$ generators $\left\{T_{r}\right\}$ in two different representations: the Solv $v_{4}$-adjoint representation $\left(\mathbb{L}_{4 r}{ }^{\hat{s}},\left(T_{r}\right)_{s}{ }^{t}\right)$ and the symplectic representation of the electric-magnetic charges $\left(\mathbb{L}_{4} M^{N},\left(T_{r}\right)_{M}{ }^{N}\right)$

$$
\begin{aligned}
& \mathbb{L}_{4}^{-1} T_{r} \mathbb{L}_{4}=\mathbb{L}_{4 r}{ }^{\hat{s}} T_{\hat{s}} ; \quad \mathbb{L}_{4}^{-1} T_{M} \mathbb{L}_{4}=\mathbb{L}_{4} M^{N} T_{N}, \\
& {\left[T_{r}, T_{s}\right]=-\left(T_{r}\right)_{s}{ }^{t} T_{t} ; \quad\left[T_{r}, T_{M}\right]=-\left(T_{r}\right)_{M}{ }^{N} T_{N} ; \quad T_{r M N} \equiv\left(T_{r}\right)_{M}{ }^{P} \mathbb{C}_{P N} .}
\end{aligned}
$$

Recalling the definition of the Lax components $Y^{A}, \mathbb{L}^{-1} \dot{\mathbb{L}}=Y^{A} T_{A}$, we can now derive their explicit form in terms of $\dot{\phi}^{i}$

$$
\begin{aligned}
Y^{0} & =2 \dot{U} ; \quad Y^{\hat{r}}=\sqrt{2} V_{s}^{\hat{r}} \dot{\phi}^{s} ; \\
Y^{M} & =\sqrt{2} \mathrm{e}^{-U} \mathbb{L}_{4 N}{ }^{M} \dot{Z}^{N} ; \\
Y^{\bullet} & =-\mathrm{e}^{-2 U}\left(\dot{a}+\mathbf{Z}^{T} \mathbb{C} \dot{\mathbf{Z}}\right)
\end{aligned}
$$

where $V_{r}^{\hat{s}} d \phi^{r}$ is the vielbein of $G_{4} / H_{4}$ in the solvable coordinates, $G_{r s}=V_{r}^{\hat{t}} V_{s}^{\hat{u}} \delta_{\hat{t} \hat{u}}$. From the Lax operator we compute the sigma model metric

$$
g_{i j} \dot{\phi}^{i} \dot{\phi}^{j}=\alpha\left(Y^{A} Y^{B} \tilde{\eta}_{A B}\right) .
$$


The normalisation coefficient $\alpha$ can be fixed by comparing with the action obtained from dimensional reduction, as given by equation (A.11) in appendix A

$$
\alpha=\frac{1}{2 \tilde{\eta}_{00}} .
$$

We must furthermore have that

$$
\tilde{\eta}_{N M}=-\tilde{\eta}_{00} \delta_{N M}, \quad \tilde{\eta}_{\bullet \bullet}=\tilde{\eta}_{00}, \quad \tilde{\eta}_{r s}=\tilde{\eta}_{00} \delta_{r s},
$$

which can always be achieved by rescalings of the generators. If we insist on the normalisations for the structure constants as given by equation (5.11) we generically loose the freedom to rescale the generators such that $\tilde{\eta}_{00}=1$. For this reason we explicitly carry factors of $\tilde{\eta}_{00}$ around in our expressions.

We can now write the explicit form of $Q_{A}=\mathbb{L}_{A}^{B} Y_{B}$

$$
\begin{aligned}
\tilde{\eta}_{00}^{-1} Q_{0} & =2 \dot{U}-\mathrm{e}^{-2 U} \mathbf{Z}^{T} \mathcal{M}_{4} \dot{\mathbf{Z}}+n a=2 M_{A D M}, \\
\tilde{\eta}_{00}^{-1} Q_{r} & =\sqrt{2}\left(\mathbb{L}_{4}\right)_{r}{ }_{r}^{\hat{s}} V_{t}^{\hat{u}} \dot{\phi}^{t} \delta_{\hat{s} \hat{u}}+2 \mathrm{e}^{-2 U}\left(\mathbf{Z}^{T} T_{r} \mathcal{M}_{4} \dot{\mathbf{Z}}\right)+T_{r M N} Z^{M} Z^{N} n, \\
\tilde{\eta}_{00}^{-1} Q_{M} & =-\sqrt{2}\left(\mathrm{e}^{-2 U} \mathcal{M}_{4 M N} \dot{Z}^{N}+\mathbb{C}_{M N} Z^{N} n\right)=\sqrt{2} \mathbb{C}_{M N} \mathcal{Q}^{N}, \\
\tilde{\eta}_{00}^{-1} Q_{\bullet} & =-n,
\end{aligned}
$$

where $\mathcal{Q}=\left(m^{I}, e_{I}\right)$ is the vector of quantized charges and, as usual we define $\mathcal{M}_{4 M N} \equiv$ $\mathbb{L}_{4 M} M^{P} \mathbb{L}_{4 N}{ }^{P}>0$. It is useful to express the Noether charges in terms of the conjugate momenta $P_{i}$ : $P_{U}, P_{r}, P_{M}, P_{\bullet}$. We know that $P_{i}=g_{i j} \dot{\phi}^{j}$, which explicitly reads (see appendix)

$$
P_{U}=2 \dot{U}, P_{r}=G_{r s} \dot{\phi}^{s}, P_{M}=-e^{-2 U} \mathcal{M}_{4 M N} \dot{Z}^{N}-\frac{1}{2} n \mathbb{C}_{M N} Z^{N}, P_{\bullet}=\frac{1}{2} n .
$$

We find, after some algebra

$$
\begin{aligned}
\tilde{\eta}_{00}^{-1} Q_{0} & =P_{U}+Z^{M} P_{M}+2 a P_{\bullet}, \\
\tilde{\eta}_{00}^{-1} Q_{r} & =\sqrt{2}\left(\mathbb{L}_{4}\right)_{r}^{\hat{s}} V_{\hat{s}}^{-1 t} P_{t}-2 P_{M} T_{r N}{ }^{M} Z^{N}, \\
\mathcal{Q}^{M} & =\mathbb{C}^{M N} P_{N}-Z^{M} P_{\bullet}, \\
\tilde{\eta}_{00}^{-1} Q_{\bullet} & =-2 P_{\bullet} .
\end{aligned}
$$

\subsection{The (fake) superpotentials $\mathcal{W}_{4}, \mathcal{W}_{3}$}

The (fake) superpotential $\mathcal{W}_{4}$ mentioned in the Introduction, is solution to the HamiltonJacobi equation associated to the autonomous system (1.1) describing the radial flow of the four-dimensional scalar fields and warp factor:

$$
\frac{1}{2}\left(\partial_{U} \mathcal{W}_{4}\right)^{2}+\partial_{r} \mathcal{W}_{4} \partial^{r} \mathcal{W}_{4}=2 \mathrm{e}^{2 U} V
$$

while $\mathcal{W}_{3} \equiv \mathcal{W}$ is solution to eq. (3.37), associated with the three-dimensional Hamiltonian system. For static solutions the relation between the superpotentials $\mathcal{W}_{4}, \mathcal{W}_{3} \equiv \mathcal{W}$ was found in [8] to be:

$$
\mathcal{W}\left(\phi^{i}\right)=\mathcal{W}_{4}\left(U, \phi^{r} ; m, e\right)+Z^{M} \mathbb{C}_{M N} \mathcal{Q}^{N}=\mathcal{W}_{4}+Z_{I} m^{I}-Z^{I} e_{I} .
$$




\subsection{The hamiltonians}

Let us review, in brief, the algorithm introduced in [48] for constructing the Hamiltonians in involution associated with the Lax equation for the $\mathrm{S} \ell(N, \mathbb{R})$-models ${ }^{11}$ (a more general construction will be given in [50]).

In order to construct the Hamiltonians, one makes use of the embedding (4.14). Let us introduce two indices

$$
\begin{aligned}
a & =0, \cdots,\left[\frac{N}{2}\right], \\
\alpha & =1, \cdots, N-2 a .
\end{aligned}
$$

We will denote the Hamiltonians $\mathcal{H}\left(Y_{A}\right)$ by $\mathfrak{h}_{a \alpha}$. The functions $\mathfrak{h}_{a \alpha}$ are then obtained by the relation

$$
\begin{aligned}
& \operatorname{det}\left\{(L-\lambda \mathbb{1})_{i j}: a+1 \leq i \leq N, 1 \leq j \leq N-a\right\} \\
& \quad=\mathcal{E}_{a 0}\left(\lambda^{N-2 a}+\sum_{\alpha=1}^{N-2 a} \mathfrak{h}_{a \alpha} \lambda^{N-2 a-\alpha}\right), \quad a=0, \cdots,\left[\frac{N}{2}\right],
\end{aligned}
$$

where $\mathcal{E}_{a 0}$ is the coefficient of the power $\lambda^{N-2 a}$. One can check that the coefficient $\mathfrak{h}_{01}$ corresponds to $\operatorname{Tr}(L)$ and is therefore zero. The Hamiltonians $\mathfrak{h}_{0 \alpha}$ are essentially the coefficients of the characteristic polynomial of $L$ and are polynomial in the $Y_{A \text {-variables. }}$ More specifically, $\mathfrak{h}_{02}$ is quadratic and corresponds to the Hamiltonian function $\mathcal{H}$ defined in (3.2). For $a \neq 0$, the $\mathfrak{h}_{a \alpha}$ are rational functions of $Y_{A}$.

\subsection{Regularity}

It is important to realise that not every geodesic corresponds to a (physically reasonable) black hole solution. We already mentioned that the solutions with $v^{2}<0$ are over-extreme. There is a convenient way to parametrise those $Q$ that correspond to regular black hole solutions. ${ }^{12}$ This is based on a theorem due to Breitenlohner, Gibbons and Maison [20]. This theorem states that all regular non-extremal black hole solutions can be transformed to the Schwarzschild solution using $G$-transformations. This theorem can be rephrased on the level of the matrix $Q$, as shown in [29]. For the Schwarschild solution, $U$ is the only scalar that is turned on. Hence we have $\mathbb{L}=\mathrm{e}^{U H_{0}}$ and $\mathbb{M}=\eta \mathrm{e}^{2 U H_{0}}$. Let us denote the Noether charge that belongs to the Schwarzschild solution by $Q_{s}$, then we have

$$
Q_{s}=2 v H_{0}
$$

In the adjoint representation we have the peculiar property that $H_{0}$ has a 5 grading structure. This means that $H_{0}$ is proportional to a diagonal matrix with only $-2,-1,0,+1,+2$

\footnotetext{
${ }^{11}$ As anticipated in the Introduction, this algorithm also works for the STU model.

${ }^{12}$ Irregular solutions are not considered physical unless the naked singularity is lightlike such that it coincides with the horizon (small black holes). These cases are still of interest since one expects that derivative corrections change the solution such as to develop a horizon to cloak the singularity.
} 
as entries. If we fix the normalisation of the generators such that $H_{0}$ contains only the $-2,-1,0,+1,+2$ as entries then

$$
Q_{s}^{5}=20 v^{2} Q_{s}^{3}-64 v^{4} Q_{s} .
$$

Since $Q$ transforms in the adjoint of $G_{3}: Q \rightarrow g Q g^{-1}$, we find that the above relation is fulfilled for all charge matrices that are connected with $Q_{s}$ and hence for all regular non-extremal black hole solutions. For $\mathrm{E}_{8}$ we have the particular property that the adjoint representation is also the fundamental representation. But for all other groups encountered in supergravity this is not the case and the particular property of these groups is that the fundamental representation has a 3 -grading of $H_{0}$; this implies the simpler equation in the fundamental

$$
Q_{s}^{3}=4 v^{2} Q_{s} .
$$

In sum, we have that regular black holes obey

$$
Q^{5}=20 v^{2} Q^{3}-64 v^{4} Q .
$$

in the adjoint and

$$
Q^{3}=4 v^{2} Q
$$

in the fundamental for all algebras of interest but $\mathrm{E}_{8}$. From this we find the elegant result that extremal regular black holes are described by nilpotent matrices for which the expansion $\mathrm{e}^{Q \tau}$ terminates after a finite number of steps leading to polynomial expressions for the entries of $\mathbb{M}$ and hence to simpler expressions for the scalars. It was conjectured in [29] that in the limit $v \rightarrow 0$ another regularity condition exists, which can be written in terms of weighted Dynkin diagrams, but we do not investigate this further.

The regularity condition also facilitates the construction of explicit solutions. If one constructs explicit solutions using the formalism of the symmetric coset matrix $\mathbb{M}$, then regularity implies

$$
\mathbb{M}=\mathbb{M}(0)\left(\mathbb{1}+\tau Q+\frac{1}{2} \tau^{2} Q^{2}\right)
$$

when $v=0$ and

$$
\mathbb{M}=\mathbb{M}(0)\left(\mathbb{1}+\frac{\sinh (2 v \tau)}{2 v} Q+\frac{\cosh (2 v \tau)-1}{4 v^{2}} Q^{2}\right) .
$$

when $v \neq 0$. Similarly in the Lax formalism of section 4, where the computations rely on the matrix $\mathcal{C}(\tau)=\mathrm{e}^{-2 \tau L_{0}}$, which expands in the same way as the matrix $\mathbb{M}$ given above.

On the level of the Hamiltonians the regularity of the solutions becomes straightforward. First we observe that the polynomial Hamiltonians are invariant under $G$. Hence, from Mazur's theorem, we deduce that the polynomial Hamiltonians take the same value as they do for the Schwarzschild solution. This means that a necessary condition for regularity is that all polynomial Hamiltonians, but the quadratic one $\left(\mathcal{H} \propto v^{2}\right)$, are zero. This condition is also sufficient. For that we observe that the polynomial Hamiltonians are the coefficients of the characteristic polynomial $P_{L}(\lambda)$

$$
P_{L}(\lambda): \quad \lambda^{N}+\mathcal{H}_{2}(L) \lambda^{N-2}+\mathcal{H}_{3}(L) \lambda^{N-3}+\ldots+\mathcal{H}_{N} .
$$


If we use the Cayley-Hamilton theorem, $P_{L}(L)=0$, together with the fact that all $\mathcal{H}_{i}=0$ with $i>2$, we find

$$
L^{N}+\mathcal{H}_{2} L^{N-2}=0 .
$$

We cannot multiply this equation with $L^{3-N}$ to find the regularity condition (5.36) since $L$ is generically not invertible (and especially not when it corresponds to a regular solution). However the above equation does imply that the eigenvalues $\lambda$ of $L$ obey

$$
\lambda^{n}+\mathcal{H}_{2} \lambda^{n-2}=0
$$

with solutions $\lambda= \pm \sqrt{\mathcal{H}_{2}}, 0$. This however implies the 3 -grading structure and hence the regularity condition $(5.36) .{ }^{13}$

\subsection{Normal forms}

The above discussion is an example of how normal forms are useful. By definition a normal form is the simplest form of $Q \in \mathfrak{g} \ominus \mathfrak{H}$ obtained under the adjoint action of $H^{*}$. Thus, the Schwarzschild solution is the normal form of all regular non-extremal solutions. Another useful aspect of normal forms comes from the fact that supersymmetry commutes with $G$ and hence the study of the supersymmetry properties of the normal form suffices to understand the supersymmetry of the general solution.

In [25] the normal form of $Q$ for a generic geodesic was derived. This is more general then the extended theorem of Mazur [20] since it applies also to extremal solutions and to singular solutions.

The theorem of [25] states that the normal form $Q_{N}$ is given by the following element of the algebra $\mathfrak{g}$ :

$$
Q_{N}=\oplus_{i=1}^{p}[\mathfrak{s l}(2) \ominus \mathfrak{s o}(1,1)] \quad \oplus_{j=1}^{q} \mathfrak{s o}(1,1) \oplus N i l .
$$

The number $p$ is the rank of the coset $H^{*} / H_{c}$, with $H_{c}$ the maximal compact subgroup of $H^{*}$. Nil is a nilpotent generator that commutes with the first part of the normal form. In [25] the focus was on solutions where Nil is absent and the generating solution is described by $p$ axion-dilaton pairs and $q$ dilatons. The reason is that these solutions are limits of non-extremal solutions with a (complex) diagonalisable initial condition. It was also noted that the known attractor solutions in $\mathcal{N}=8$ supergravity could be understood from the normal form without $N i l$. Regularity and the absence of naked singularities implies that none of the $p$ axion-dilaton pairs can have negative velocity squared. Hence extremal solutions are obtained by taking the $p$ axion-dilaton pairs to be lightlike and to truncate the $q$ decoupled dilaton scalars. The susy properties of the various solutions for the $\mathcal{N}=8$ theory (and the related $\mathcal{N}=2 \mathrm{STU}$ model) have been investigated using the normal form in [25], see also [29-32] for more recent work.

\footnotetext{
${ }^{13}$ The regularity condition (5.36) implies that the solution is on the Schwarzschild orbit, although we have not yet proven this in that direction. This is however straightforward since the eigenvalues of $L$ have to be $0, \sqrt{\mathcal{H}_{2}}$ and $-\sqrt{\mathcal{H}_{2}}$. The multiplicities of the eigenvalues is deduced using $\operatorname{Tr} L=0$ and $\operatorname{Tr} L^{2}=2 \mathcal{H}_{2}$. Indeed 0 has multiplicity $N-2$ and the other two eigenvalues each have multiplicity 1 , showing that it is the Schwarzschild solution.
} 


\subsection{The dilatonic black hole}

Consider the Einstein-Maxwell-dilaton action

$$
S=\int \sqrt{|g|}\left(\frac{1}{2} \mathcal{R}-\frac{1}{2}(\partial \phi)^{2}-\frac{1}{4} \mathrm{e}^{a \phi} F^{2}\right) .
$$

This is probably the easiest theory that can describe black holes with scalar hair. When the dilaton coupling $a$ obeys $a^{2}=6$ then the action (5.43) is just the circle reduction of pure gravity in $d=5 .{ }^{14}$ This model can therefore be embedded in many supergravity theories (so does the $a^{2}=2$ theory). The reduction over time leads to the $\mathrm{S} \ell(3, \mathbb{R}) / \mathrm{SO}(2,1)$ coset. The black hole solutions of this theory have been considered by many authors before $[19,28,33,61]$. Here we redo the analysis in our framework because it nicely illustrates our formalism.

\subsubsection{The $\frac{\mathrm{S} \ell(3, \mathbb{R})}{\mathrm{SO}(2,1)}$ sigma model}

We define the coset element as in equation (5.18) and consider the following fundamental representation for the generators

$$
\begin{aligned}
& T_{0}=\frac{1}{2}\left(\begin{array}{ccc}
1 & 0 & 0 \\
0 & 0 & 0 \\
0 & 0 & -1
\end{array}\right), \quad T_{\bullet}=\left(\begin{array}{lll}
0 & 0 & 1 \\
0 & 0 & 0 \\
0 & 0 & 0
\end{array}\right), \quad T_{\phi}=\frac{1}{2 \sqrt{3}}\left(\begin{array}{ccc}
1 & 0 & 0 \\
0 & -2 & 0 \\
0 & 0 & 1
\end{array}\right), \\
& T_{1}=\left(\begin{array}{lll}
0 & 0 & 0 \\
0 & 0 & 1 \\
0 & 0 & 0
\end{array}\right), \quad T_{2}=\left(\begin{array}{lll}
0 & 1 & 0 \\
0 & 0 & 0 \\
0 & 0 & 0
\end{array}\right) \text {. }
\end{aligned}
$$

The notation in the above expressions is as before, where $M$ runs over $\{1,2\}$ and, since the solvable algebra in $d=4$ is trivial, we have denoted $T_{r}=T_{\phi}$. From these solvable generators we can construct the coset generators $K_{A}$ via $K_{A}=\frac{1}{2}\left(T_{A}+\eta T_{A}^{T} \eta\right)$ with $\eta$ given by

$$
\eta=\left(\begin{array}{ccc}
1 & 0 & 0 \\
0 & -1 & 0 \\
0 & 0 & 1
\end{array}\right) \text {. }
$$

This allows us to compute the coset metric $\tilde{\eta}_{A B}=\operatorname{Tr}\left(K_{A} K_{B}\right)$

$$
\tilde{\eta}=\operatorname{diag}\left(\tilde{\eta}_{00}, \tilde{\eta}_{\phi \phi}, \tilde{\eta}_{11}, \tilde{\eta}_{22}, \tilde{\eta}_{\bullet \bullet}\right)=\operatorname{diag}\left(\frac{1}{2}, \frac{1}{2},-\frac{1}{2},-\frac{1}{2},-\frac{1}{2}\right) .
$$

This coset metric indeed satisfies the normalisations (5.25). The coset representative (5.18) is explicitly given by $\mathbb{L}=\mathrm{e}^{-a T_{\bullet}} \mathrm{e}^{\sqrt{2} Z^{M} T_{M}} \mathrm{e}^{2 \sqrt{2} \phi T_{\phi}} \mathrm{e}^{2 U T_{0}}$. In components this is

$$
\mathbb{L}=\left(\begin{array}{ccc}
\mathrm{e}^{U+\frac{\sqrt{2}}{\sqrt{3}} \phi} & \sqrt{2} \mathrm{e}^{\frac{-2 \sqrt{2}}{\sqrt{3}} \phi} Z^{2} & \mathrm{e}^{-U+\frac{\sqrt{2}}{\sqrt{3}} \phi}\left(-a+Z^{1} Z^{2}\right) \\
0 & \mathrm{e}^{\frac{-2 \sqrt{2}}{\sqrt{3}} \phi} & \sqrt{2} \mathrm{e}^{-U+\frac{\sqrt{2}}{\sqrt{3}} \phi} Z^{1} \\
0 & 0 & \mathrm{e}^{-U+\frac{\sqrt{2}}{\sqrt{3}} \phi}
\end{array}\right) .
$$

\footnotetext{
${ }^{14}$ In the language of the general sigma model treated in the appendix we have $G_{r s}=1, \mu_{I J}=\mathrm{e}^{2 \sqrt{3} \phi}$ and $\nu_{I J}=0$.
} 
From this we can compute the Lax operator

$$
L=\left(\begin{array}{ccc}
\frac{1}{2} Y^{0}+\frac{1}{2 \sqrt{3}} Y^{\phi} & \frac{1}{2} Y^{2} & \frac{1}{2} Y^{\bullet} \\
-\frac{1}{2} Y^{2} & -\frac{1}{\sqrt{3}} Y^{\phi} & \frac{1}{2} Y^{1} \\
\frac{1}{2} Y^{\bullet} & -\frac{1}{2} Y^{1} & -\frac{1}{2} Y^{0}+\frac{1}{2 \sqrt{3}} Y^{\phi}
\end{array}\right),
$$

with

$$
\begin{aligned}
& Y^{0}=2 \dot{U}, \quad Y^{\phi}=\sqrt{2} \dot{\phi}, \\
& Y^{1}=\sqrt{2} \mathrm{e}^{-U+\sqrt{6} \phi} \dot{Z}^{1}, \\
& Y^{2}=\sqrt{2} \mathrm{e}^{-U-\sqrt{6} \phi} \dot{Z}^{2}, \\
& Y^{\bullet}=-\mathrm{e}^{-2 U}\left(\dot{a}+Z^{2} \dot{Z}^{1}-Z^{1} \dot{Z}^{2}\right) .
\end{aligned}
$$

\subsubsection{The solutions}

In order to investigate regular solutions of this model, we first show how the regularity condition (5.34) can be simplified. As the initial Lax operator $L_{0}=L(\tau=0)$ is an element of the $\mathrm{S} \ell(3)$ algebra, it obeys the following identity

$$
L_{0}^{3}=\frac{1}{2} \operatorname{Tr}\left(L_{0}^{2}\right) L_{0}+\frac{1}{3} \operatorname{Tr}\left(L_{0}^{3}\right) \mathbb{1} .
$$

From (5.34), one thus sees that the condition for having regular solutions is simply

$$
\operatorname{Tr}\left(L_{0}^{3}\right)=0 .
$$

Of course, this is the $\mathrm{S} \ell(3)$-version of the statement that all polynomial Hamiltonians, but the quadratic one, have to vanish for regular solutions (see the discussion around equation (5.39)). Practically this means that the dilaton charge can be written in terms of the mass and the electric-magnetic charges, something that was known before [61]. In the extremal case one has

$$
\operatorname{Tr}\left(L_{0}^{2}\right)=0 \quad \Rightarrow \quad v=0,
$$

we then immediately find that $L_{0}$ is nilpotent of degree $3: L_{0}^{3}=0$. This allows only 2 arbitrary charges since $\operatorname{Tr}\left(L_{0}^{3}\right)=\operatorname{Tr}\left(L_{0}^{2}\right)=0$ give 2 conditions for 4 charges.

In order to find solutions using the integration algorithms, we will use a specific parametrization for the initial condition $L_{0}$, inspired from [61], that solves the regularity condition

$$
L_{0}=\left(\begin{array}{ccc}
\frac{\alpha}{2} & \frac{-1}{2} \sqrt{\frac{\alpha^{3}-4 v^{2} \alpha}{\alpha-\beta}} & 0 \\
\frac{1}{2} \sqrt{\frac{\alpha^{3}-4 v^{2} \alpha}{\alpha-\beta}} & \frac{-1}{2}(\alpha+\beta) & \frac{-1}{2} \sqrt{\frac{\beta^{3}-4 v^{2} \beta}{\beta-\alpha}} \\
0 & \frac{1}{2} \sqrt{\frac{\beta^{3}-4 v^{2} \beta}{\beta-\alpha}} & \frac{\beta}{2}
\end{array}\right) .
$$

We can choose the gauge in which $\mathbb{L}_{3}(0)=\mathbb{1}$. This implies that $U(0)=1$, which is the usual coordinate choice, $Z^{M}(0)=a(0)=0$, which can be consistently obtained, when $n=0$, by shifts of the axions. Only the choice $\phi(0)=0$ is a restriction. However, if we use the dilatation symmetry in 4 dimensions:

$$
\phi \rightarrow \phi+c, \quad F_{\tau t} \rightarrow F_{\tau t} \mathrm{e}^{\sqrt{6} c}, \quad F_{\theta \phi} \rightarrow F_{\theta \phi} \mathrm{e}^{-\sqrt{6} c} .
$$


we can generate the solutions with $\phi(0) \neq 0$.

Let us solve first for the non-extremal solutions. The initial Lax operator is given by (5.53). We present the solution for the initial condition $\phi(0)=0$. In terms of the two functions $B(\tau), C(\tau)$

$$
\begin{aligned}
& B(\tau)=\frac{\alpha\left(4 v^{2}-\beta^{2}\right)+\beta\left(-4 v^{2}+\alpha \beta\right) \cosh (2 v \tau)+2 v(\alpha-\beta) \beta \sinh (2 v \tau)}{4 v^{2}(\alpha-\beta)}, \\
& C(\tau)=\frac{\left(-4 v^{2}+\alpha^{2}\right) \beta+\alpha\left(4 v^{2}-\alpha \beta\right) \cosh (2 v \tau)+2 v \alpha(-\alpha+\beta) \sinh (2 v \tau)}{4 v^{2}(\alpha-\beta)}
\end{aligned}
$$

the solution reads

$$
\mathrm{e}^{2 U}=(B C)^{-1 / 2}, \quad \mathrm{e}^{\phi}=\left(B^{-1} C\right)^{-\frac{\sqrt{3}}{2 \sqrt{2}}} .
$$

In the extremal limit, defined by $v=0$, the corresponding solution is given by equation (5.56), where $B$ and $C$ now read

$$
B(\tau)=1+\beta \tau+\frac{\alpha \beta^{2}}{2(\alpha-\beta)} \tau^{2}, \quad C(\tau)=1-\alpha \tau-\frac{\alpha^{2} \beta}{2(\alpha-\beta)} \tau^{2} .
$$

We can take the limit where either the electric or the magnetic charge vanishes. Then the solution simplifies considerably and the regular horizon collapses to coincide with the black hole singularity: the solution becomes a small black hole. One can readily check that the nilpotency degree of $L_{0}$ becomes 2 instead of 3 in this limit.

With $\mathbb{L}_{3}(0)=\mathbb{1}$ we have $Q=L_{0}$. Through equations (5.26), this allows us to give a physical interpretation to $\alpha$ and $\beta$

$$
\begin{aligned}
& Q_{0}=\frac{1}{4}(\alpha-\beta)=M \\
& Q_{\bullet}=0=-\frac{1}{2} n \\
& Q_{1}=\frac{1}{2} \sqrt{\frac{\beta^{3}-4 v^{2} \beta}{\beta-\alpha}}=-\frac{1}{\sqrt{2}} \mathcal{Q}_{\text {electric }}, \\
& Q_{2}=\frac{1}{2} \sqrt{\frac{\alpha^{3}-4 v^{2} \alpha}{\alpha-\beta}}=\frac{1}{\sqrt{2}} \mathcal{Q}_{\text {magnetic }}, \\
& Q_{\phi}=\frac{\sqrt{3}}{2}(\alpha+\beta) .
\end{aligned}
$$

\subsubsection{The normal form}

Since the scalar manifold in three dimensions is $\mathrm{S} \ell(3, \mathbb{R}) / \mathrm{SO}(2,1)$, the normal form of $L_{0}$ should be given by

$$
L_{0} \in[\mathfrak{s l}(2) \ominus \mathfrak{s o}(1,1)] \oplus \mathfrak{s o}(1,1) \oplus N i l .
$$

For extremal solutions the decoupled $\mathfrak{s o}(1,1)$ should be zero. One verifies that the $\frac{\mathfrak{s l}(2)}{\mathfrak{s o}(2,1)}$ part has nilpotency degree 2 in the fundamental. This implies that we need the extra nilpotent generator $\mathrm{Nil}$ of degree 3 in order to have a regular solution. Indeed, the solution 
without $N i l$ corresponds to the small black hole solution that has either vanishing magnetic or electric charge. Furthermore, if one also truncates the lightlike charges in the $\frac{\mathfrak{s l}(2)}{\mathfrak{s o}(2,1)}$ part, such that one is left with the Nil generator, only then one recovers the double extremal solution. This is the solution for which the dilaton is constant everywhere since its initial value is at the minimum of the black hole potential and its initial velocity is zero. This occurs when $\alpha=-\beta$.

\subsubsection{Hamiltonian viewpoint}

Following the procedure outlined in section 3, we can construct the Hamiltonians in involution needed for integrability, two of which depend on the $Y^{15}$

$$
\begin{aligned}
\mathcal{H}_{1}(Y)= & -\frac{1}{4}\left(Y^{0}\right)^{2}+\frac{1}{4}\left(Y^{1}\right)^{2}+\frac{1}{4}\left(Y^{2}\right)^{2}-\frac{1}{4}\left(Y^{\phi}\right)^{2} \\
\mathcal{H}_{2}(Y)= & \frac{1}{8} Y^{0}\left(\left(Y^{2}\right)^{2}-\left(Y^{1}\right)^{2}\right)-\frac{1}{8 \sqrt{3}} Y^{\phi}\left(\left(Y^{1}\right)^{2}+\left(Y^{2}\right)^{2}\right) \\
& +\frac{1}{12 \sqrt{3}}\left(Y^{\phi}\right)^{3}-\frac{1}{4 \sqrt{3}}\left(Y^{0}\right)^{2} Y^{\phi} .
\end{aligned}
$$

The first of these, $\mathcal{H}_{1}\left(Y^{A}\right)$ corresponds to $\mathcal{H}=-\frac{1}{2} Y_{A} Y^{A}$ and vanishes for extremal solutions. The second Hamiltonian, $\mathcal{H}_{2}\left(Y^{A}\right)$, is cubic and proportional to $\operatorname{Tr}\left(L_{0}^{3}\right)$. It vanishes for regular solutions.

The proof of integrability requires two extra Hamiltonians, that now depend on the charges $Q_{A}$. In principle they are obtained by making the substitution $Y^{A} \rightarrow Q_{A}$ in $\mathcal{H}_{1}\left(Y^{A}\right), \mathcal{H}_{2}\left(Y^{A}\right)$. This however leads to rather complicated expressions. Since we are considering vanishing Taub-NUT charge here, we can adopt a more practical approach. We simply choose the two electro-magnetic charges as Hamiltonians

$$
\mathcal{H}_{3}\left(Q_{A}\right)=Q_{1}, \quad \mathcal{H}_{4}\left(Q_{A}\right)=Q_{2}
$$

These still Poisson-commute with the $Y^{A}$ variables and hence with $\mathcal{H}_{1}$ and $\mathcal{H}_{2}$. Moreover, for vanishing Taub-NUT charge, they also Poisson-commute among themselves since the electric and magnetic charges close a Heisenberg algebra with the Taub-NUT charge. For vanishing Taub-NUT charge this Heisenberg algebra degenerates and $\mathcal{H}_{3}$ and $\mathcal{H}_{4}$ commute. On-shell, the Hamiltonians take on constant values $\mathcal{H}_{i}(t) \equiv h_{i}$. The interpretations of these Hamiltonians is now straightforward. The first Hamiltonian $h_{1}$ corresponds to the extremality parameter. The second Hamiltonian keeps track of the regularity condition; if $h_{2}=0\left(h_{2} \neq 0\right)$ the solution is regular (singular). The third and fourth hamiltonian are proportional to the magnetic and electric charge. Hence, regular extremal solutions are parameterized by two numbers $\left(Q_{\text {electric }}, Q_{\text {magnetic }}\right)$ and regular non-extremal solutions are parameterized by three numbers $\left(Q_{\text {electric }}, Q_{\text {magnetic }}\right)$ and $h_{1}$.

\footnotetext{
${ }^{15}$ Note that we are restricting ourselves here to the case where the Taub-NUT charge vanishes. In case one allows for a non-zero Taub-NUT charge, the procedure of section 3 leads to an extra Hamiltonian, as would be required by integrability in that case. This extra Hamiltonian is a rational function of the $Y^{A}$ and corresponds to a Casimir, in the terminology of section 3.3. For vanishing Taub-NUT charge, this extra Hamiltonian is however not present.
} 
Recalling the sigma-model

$$
\mathcal{L}=\frac{1}{2} g_{i j} \dot{\phi}^{i} \dot{\phi}^{j}=\dot{U}^{2}+\frac{1}{2} \dot{\phi}^{2}-\frac{1}{2} \mathrm{e}^{-2 U+2 \sqrt{3} \phi}\left(\dot{Z}^{1}\right)^{2}-\frac{1}{2} \mathrm{e}^{-2 U-2 \sqrt{3} \phi}\left(\dot{Z}^{2}\right)^{2},
$$

where the generalised coordinates are $U, \phi, Z^{1}, Z^{2}$, the conjugate momenta are given by

$$
P_{U}=2 \dot{U}, \quad P_{\phi}=\dot{\phi}, \quad P_{1}=-\mathrm{e}^{-2 U+2 \sqrt{3} \phi} \dot{Z}^{1}, \quad P_{2}=-\mathrm{e}^{-2 U-2 \sqrt{3} \phi} \dot{Z}^{2} .
$$

The four non-vanishing Hamiltonians in (5.64) and (5.66) may then be expressed in terms of the coordinates and momenta as follows

$$
\begin{aligned}
\mathcal{H}_{1}= & -\frac{1}{4} P_{U}^{2}-\frac{1}{2} P_{\phi}^{2}+\frac{1}{2} \mathrm{e}^{2 U-2 \sqrt{3} \phi} P_{1}^{2}+\frac{1}{2} \mathrm{e}^{2 U+2 \sqrt{3} \phi} P_{2}^{2}, \\
\mathcal{H}_{2}= & -\frac{1}{2 \sqrt{6}} P_{U}^{2} P_{\phi}+\frac{1}{3 \sqrt{6}} P_{\phi}^{3}-\frac{1}{4} \mathrm{e}^{2 U-2 \sqrt{3} \phi} P_{U} P_{1}^{2}-\frac{1}{2 \sqrt{6}} \mathrm{e}^{2 U-2 \sqrt{3} \phi} P_{\phi} P_{1}^{2} \\
& +\frac{1}{4} \mathrm{e}^{2 U+2 \sqrt{3} \phi} P_{U} P_{2}^{2}-\frac{1}{2 \sqrt{6}} \mathrm{e}^{2 U+2 \sqrt{3} \phi} P_{\phi} P_{2}^{2}, \\
\mathcal{H}_{3}= & \frac{\sqrt{2}}{2} P_{1}, \quad \mathcal{H}_{4}=\frac{\sqrt{2}}{2} P_{2} .
\end{aligned}
$$

We can write the momenta $P_{A}$ in terms of the coordinates by solving the 4 equations

$$
h_{i}=\mathcal{H}_{i}\left(P_{A},\left\{U, \phi, Z^{1}, Z^{2}\right\}\right)
$$

in terms of the $P_{A}$

$$
P_{A}=f_{A}\left(U, \phi, Z^{1}, Z^{2}\right),
$$

when the Jacobian $J=\operatorname{det}\left(\frac{\partial \mathcal{H}_{i}}{\partial P_{j}}\right)$ is non-zero. For the example at hand we find

$$
\begin{aligned}
J= & -\frac{1}{8} e^{2 U-2 \sqrt{3} \phi} P_{1}^{2} P_{\phi}+\frac{1}{8 \sqrt{6}} e^{2 U-2 \sqrt{3} \phi} P_{1}^{2} P_{U}+\frac{1}{8} e^{2 \sqrt{3} \phi+2 U} P_{2}^{2} P_{\phi} \\
& +\frac{1}{8 \sqrt{6}} e^{2 \sqrt{3} \phi+2 U} P_{2}^{2} P_{U}-\frac{\sqrt{6}}{8} P_{\phi}^{2} P_{U}+\frac{1}{8 \sqrt{6}} P_{U}^{3} .
\end{aligned}
$$

From this explicit expression one can infer that the regularity condition $h_{2}=0$ can not be satisfied when $J=0$. Hence, for physical solutions $h_{2}=0$, it makes sense to define the first order equation (5.71).

It then turns out that solving explicitly for the $P_{A}$ in terms of the coordinates is the easiest for the extremal solutions $h_{1}=0$. The result is

$$
\begin{aligned}
& P_{U}=\frac{1}{2} \mathrm{e}^{\sqrt{3} \phi+U}\left(h_{4}^{3 / 2}+\mathrm{e}^{-\frac{4}{\sqrt{3}} \phi} h_{3}^{2 / 3}\right)^{3 / 2}, \\
& P_{\phi}=\frac{\sqrt{6}}{4} \mathrm{e}^{U}\left(\mathrm{e}^{\sqrt{3} \phi} h_{4}^{2 / 3}-\mathrm{e}^{-\frac{1}{\sqrt{3}} \phi} h_{3}^{2 / 3}\right) \sqrt{h_{4}^{2 / 3}+\mathrm{e}^{-\frac{4}{\sqrt{3}} \phi} h_{3}^{2 / 3}} .
\end{aligned}
$$

We can explicitly integrate this to find the $4 d$ fake superpotential

$$
\mathcal{W}_{4}(U, \phi) \equiv 2 \mathrm{e}^{U} W(\phi), \quad W(\phi)=\frac{1}{2}\left(\mathrm{e}^{\frac{2}{\sqrt{3}} \phi} h_{4}^{2 / 3}+\mathrm{e}^{-\frac{2}{\sqrt{3}} \phi} h_{3}^{2 / 3}\right)^{3 / 2}
$$


As expected the fake superpotential factorizes with the black hole warp factor $U$ [19].

In the non-extremal case the expressions for $P_{A}$ in terms of the coordinates are page filling unless we take either the magnetic or electric charge equal to zero. When the electric charge is zero we find

$$
\begin{aligned}
& P_{U}=-\frac{1}{2} \sqrt{\mathrm{e}^{2 \sqrt{3} \phi+2 U} h_{4}^{2}-16 h_{1}}, \\
& P_{\phi}=-\frac{\sqrt{6}}{4} \sqrt{\mathrm{e}^{2 \sqrt{3} \phi+2 U} h_{4}^{2}-16 h_{1}},
\end{aligned}
$$

where $h_{1}<0$. The $4 d$ fake superpotential then becomes

$$
\begin{aligned}
\mathcal{W}_{4}(U, \phi)= & \sqrt{\mathrm{e}^{2 \sqrt{3} \phi+2 U} h_{4}^{2}-16 h_{1}}+\sqrt{-h_{1}} \log \left(-16 h_{1}+4 \sqrt{-h_{1}} \sqrt{\mathrm{e}^{2 \sqrt{3} \phi+2 U} h_{4}^{2}-16 h_{1}}\right) \\
& -4 \sqrt{-h_{1}}(U+\sqrt{3} \phi) .
\end{aligned}
$$

This indeed coincides with the expressions found in [19] and in the limit $h_{1} \rightarrow 0$ it collapses to the single charge expression for the extremal superpotential (5.75)

$$
\mathcal{W}_{4}(U, \phi)=\mathrm{e}^{U} \mathrm{e}^{\sqrt{3} \phi} h_{4}
$$

\section{Discussion}

Let us summarize the main results obtained in this paper. We have established new insights in the solvability and integrability of the geodesic equations of motion on symmetric coset spaces that appear as sigma models of supergravity theories that are reduced over the timelike direction.

Concerning the solvability we have presented a recursive but closed formula for the coset representative describing a generic geodesic solution to equation (4.24). This extends the existing results that only supply a general formula for the Lax operator $[34,37,40,41,48,54-56]$. Finding the coset representative still implied solving first order differential equations in that approach. It is not always fully appreciated in the literature that the solvability was not yet established for the coset representative $\mathbb{L}$. This is due to the fact that the solution for the symmetric combination $\mathbb{M}=\mathbb{L} \eta \mathbb{L}^{T}$ can be obtained from exponentiating a Lie algebra element. However, in order to extract $\mathbb{L}$ in the solvable parametrisation, which defines the physical fields [62], from this symmetric combination requires solving an involved equation for the compensator. This problem is circumvented by the algorithm we propose.

Concerning the issue of formal integrability we have proven in a constructive way that the second order equations of motion, i.e. the full autonomous Hamiltonian system, are integrable in the sense of Liouville, once the same property has been established for the first order Lax pair problem. This means that we have proven the existence of $n$ constants of motion that mutually Poisson-commute, where $n$ is the dimension of the symmetric space, starting from those associated with the Lax pair problem. This extends the previous result which had proven Liouville integrability of the first order differential equations obeyed by 
the tangent to the geodesic. These results solve an open-standing question about the existence of a fake superpotential for black hole solutions. The fake superpotential description is the supergravity-way of describing Hamilton-Jacobi integrability and since Liouville integrability implies the latter we have proven the (local) existence of a fake superpotential for all stationary, spherically symmetric, black hole solutions to symmetric supergravity theories. The obstruction to a global existence of $\mathcal{W}$ is due to the existence of points in which the Jacobian $\operatorname{det}\left(\partial h_{i} / \partial P_{j}\right) \neq 0$ vanishes. We have proven in an explicit example that this Jacobian is always non-vanishing in the subspace spanned by regular solutions, thus ensuring therein a global existence of $\mathcal{W}$. We expect this to be the case for generic symmetric geometries, though a general proof is missing.

We also presented an explicit construction for the $n$ constants of motion leaning on earlier obtained results [48]. The constants are divided in constants that depend solely on $Y, \mathcal{H}_{\alpha}(Y)$, and constants that depend solely on $Q, \mathcal{H}_{a}(Q)$, where $Y$ are the Lax components and the $Q$ are the Noether charge components in the solvable directions. The constants that depend on $Y$ can be divided in two sets: $\mathcal{H}_{\alpha}(Y)=\left\{\mathcal{H}_{\ell}(Y), \mathcal{H}_{a}(Y)\right\}$, where the $\mathcal{H}_{\ell}(Y)$ correspond to Casimirs. Among the $\mathcal{H}_{\alpha}(Y)$, there are a certain number of polynomial constants. These have a simple interpretation. One of them corresponds to the geodesic velocity, which is proportional to the extremality parameter ST. The remaining polynomial $\mathcal{H}_{\alpha}(Y)$ then keep track of the regularity of the solution: they have to vanish for a regular solution. Among the remaining $\mathcal{H}_{\alpha}(Y)$, there is one constant that corresponds to the TaubNUT charge. So far we have been unable to give a physical interpretation to the remaining constants in the set $\mathcal{H}_{\alpha}(Y)$. The constants $\mathcal{H}_{a}(Q)$ are functions of the electro-magnetic charges $\left(e^{I}, m_{I}\right)$ and have no easy interpretation. However, for most cases of interest the Taub-NUT charge vanishes and then we have shown that one can replace $\mathcal{H}_{a}(Q)$ for the pure electro-magnetic charges, making the physical interpretation of all constants, but the remaining ones in $\mathcal{H}_{\alpha}(Y)$, clear. We have explicitly worked this out for the dilatonic black holes that arise in Kaluza-Klein theories since this is the easiest possible example to demonstrate our findings with. This example has only one non-polynomial $\mathcal{H}(Y)$, which is the Taub-NUT charge, so that we were unable to learn about the physical meaning of other possible non-polynomial Hamiltonians from this example. We anticipate to investigate the Hamiltonians in more involved models, such as the STU model, which should make the physical interpretation clear.

Finally our analysis on Liouville integrability can have a bearing on the quantum description of black holes along the lines of $[13,27]$. In these papers the authors developed a quantum description of black hole based on the quantization of the radial evolution of the scalar fields and the warp factor. The resulting wave function was suggested to have, in the semi-classical limit, the following form:

$$
\Psi\left(\phi^{i}\right) \propto \exp \left(i \mathcal{W}\left(\phi^{i}\right)\right)=\exp \left(i \mathcal{W}_{4}\left(U, \phi^{r}\right)+i\left(Z_{I} m^{I}-Z^{I} e_{I}\right)\right)
$$

In this picture the $n$ Hamiltonians in involutions, which we have been dealing with in the present paper, will provide a complete set of commuting observables, in terms of which to completely characterize the black hole state. 
Understanding the physical meaning of these Hamiltonians is therefore an important step in order to understand the physics of black holes in supergravity.

\section{Acknowledgments}

W.C. is supported in part by the Natural Sciences and Engineering Research Council (NSERC) of Canada. The work of M.T. is supported by the government grant PRIN 2007. T.V.R. is supported by the Göran Gustafsson Foundation. J.R. and T.V.R. also like to thank the Politecnico di Torino for its hospitality. The work of A.S. was partially supported by the RFBR Grants No. $09-02-12417$ - ofi_m, 09-02-00725 - a, $09-02-91349-$ NNIO_a; DFG grant No 436 RUS/113/669, and the Heisenberg-Landau Program.

\section{A The sigma model in $d=3+0$}

In this section we closely follow the seminal paper [20].

Consider the action (5.1). The equations of motion for the vectors $B^{I}$ are

$$
\mathrm{d}\left(\mu_{I J} \star \mathrm{d} B^{J}-\nu_{I J} \mathrm{~d} B^{J}\right)=0,
$$

such that, locally, we can introduce the dual potentials $C_{I}$

$$
\mu_{I J} \star \mathrm{d} B^{J}-\nu_{I J} \mathrm{~d} B^{J}=\mathrm{d} C_{I}
$$

The set of field strengths $(\mathrm{d} B, \mathrm{~d} C)$ are not independent and obey the following duality relation

$$
\left(\begin{array}{l}
\mathrm{d} B \\
\mathrm{~d} C
\end{array}\right)=\mathbb{C} \mathcal{M}_{4} \star\left(\begin{array}{l}
\mathrm{d} B \\
\mathrm{~d} C
\end{array}\right),
$$

where

$$
\mathbb{C}=\left(\begin{array}{cc}
0 & -\mathbb{1} \\
+\mathbb{1} & 0
\end{array}\right), \quad \mathcal{M}_{4}=\left(\begin{array}{cc}
\mu+\nu \mu^{-1} \nu & \nu \mu^{-1} \\
\mu^{-1} \nu & \mu^{-1}
\end{array}\right)
$$

Let us now reduce the action over the timelike direction using the ansatz (5.2). The reduced action then becomes

$$
\begin{aligned}
S_{3}=\int\left(\frac{1}{2} \star\right. & R_{3}-\star \mathrm{d} U \wedge \mathrm{d} U+\frac{1}{4} \mathrm{e}^{4 U} \star F_{K K} \wedge F_{K K}-\frac{1}{2} G_{r s} \star \mathrm{d} \phi^{r} \wedge \mathrm{d} \phi^{s} \\
& +\frac{1}{2} \mu_{I J} \mathrm{e}^{-2 U} \star \mathrm{d} Z^{I} \wedge \mathrm{d} Z^{J}-\frac{1}{2} \mathrm{e}^{2 U} \mu_{I J} \star\left(\tilde{G}^{I}+Z^{I} F_{K K}\right) \wedge\left(\tilde{G}^{J}+Z^{J} F_{K K}\right) \\
& \left.-\nu_{I J}\left(\tilde{G}^{I}+Z^{I} F_{K K}\right) \wedge \mathrm{d} Z^{J}\right)
\end{aligned}
$$

The vectors $A_{K K}$ and $\tilde{B}^{I}$ can be dualised to scalars $\chi$ and $Z_{I}$ by adding them as Lagrange multipliers to the action that ensure the Bianchi identities

$$
S_{3}^{\prime}=S_{3}+\chi \mathrm{d} F_{K K}+Z_{I} \mathrm{~d} \tilde{G}^{I}
$$


Varying the action $S_{3}^{\prime}$ with respect to $F_{K K}$ and $\tilde{G}^{I}$ gives the equations of motion

$$
\begin{aligned}
\mathrm{d} Z_{J} & =-\mathrm{e}^{2 U} \star \mu_{I J}\left(\tilde{G}^{I}+Z^{I} F_{K K}\right)-\nu_{I J} \mathrm{~d} Z^{I}, \\
\mathrm{~d} \chi & =\frac{1}{2} \mathrm{e}^{4 U} \star F_{K K}+Z^{I} \mathrm{~d} Z_{I} .
\end{aligned}
$$

One can verify that the scalars $Z_{I}$ coincide with the timelike components of the dual potentials $C_{I}$

$$
C_{I}=\tilde{C}_{I}+Z_{I}\left(\mathrm{~d} t+A_{K K}\right)
$$

where $\tilde{C}_{I}$ is a vector in $d=3$.

Dualisation of the action $S_{3}$ is equivalent to eliminating $F_{K K}$ and $\tilde{G}^{I}$ from the action $S_{3}^{\prime}$ using the two identities (A.7), (A.8). If we furthermore make the field redefinition

$$
2 \chi=a+Z^{I} Z_{I},
$$

(such that $a$ is a symplectic invariant scalar) we find the action ${ }^{16}$

$$
\begin{gathered}
S_{3}=\int\left(\frac{1}{2} \star R_{3}-\star \mathrm{d} U \wedge \mathrm{d} U-\frac{1}{2} G_{r s} \star \mathrm{d} \phi^{r} \wedge \mathrm{d} \phi^{s}+\frac{1}{2} \mathrm{e}^{-2 U} \star \mathrm{d} \mathbf{Z}^{T} \wedge \mathcal{M}_{4} \mathrm{~d} \mathbf{Z}\right. \\
\left.-\frac{1}{4} \mathrm{e}^{-4 U} \star\left(\mathrm{d} a+\mathbf{Z}^{T} \mathbb{C} \mathrm{d} \mathbf{Z}\right) \wedge\left(\mathrm{d} a+\mathbf{Z}^{T} \mathbb{C d} \mathbf{Z}\right)\right) .
\end{gathered}
$$

In the main text we have used the notation

$$
\mathbf{Z} \equiv\left(Z^{M}\right)=\left(Z^{I}, Z_{I}\right) .
$$

In a compact notation we write

$$
S_{3}=\int \frac{1}{2} \star R_{3}-\frac{1}{2} g_{i j}(\phi) \star \mathrm{d} \phi^{i} \wedge \mathrm{d} \phi^{j}
$$

\section{B Conventions for charges and mass}

As usual we define charges via Gauss' law, for which we integrate a closed 3-form over the spatial dimensions:

$$
\begin{aligned}
\mathcal{Q}^{I}=m^{I} & =\frac{1}{4 \pi} \int_{3} \mathrm{~d} G^{I}=\frac{1}{4 \pi} \int_{S^{2}} G^{I}, \\
\mathcal{Q}_{I}=e_{I} & =\frac{1}{4 \pi} \int_{3} \mathrm{~d} F_{I}=\frac{1}{4 \pi} \int_{S_{2}} F_{I}, \\
n & =\frac{1}{4 \pi} \int_{3} \mathrm{~d} F_{K K}=\frac{1}{4 \pi} \int_{S_{2}} F_{K K},
\end{aligned}
$$

where

$$
F_{I}=\mathrm{d} C_{I}=\mu_{I J} \star G^{J}-\nu_{I J} G^{J} .
$$

\footnotetext{
${ }^{16}$ The sigma model metric corresponds to a Lorentzian version of the metric obtained in [42], where the similar problem was considered for time-dependent solutions, i.e. Euclidean sigma models. Once explicit solutions for the scalar fields have been obtained, one can uplift these to four dimensions using similar uplifting formula as developed in [42].
} 
We have denoted magnetic charges by $m^{I}$, electric charges by $e_{I}$ and the Taub-NUT charge by $n$. There is a subtlety in the definition of the electric and magnetic charge in a spacetime for which $n \neq 0$. In that case the integrals that define the $\mathcal{Q}$ 's are dependent on the radial coordinate and hence not constants. ${ }^{17}$ The definition that gives constant charges, when $n \neq 0$ can, for instance, be derived using Noethers theorem and is given in the main text. From the formulas of appendix A we find

$$
\begin{aligned}
\mathcal{Q}^{N} & =\mathrm{e}^{-2 U}(\mathbb{C} M \dot{\mathbf{Z}})^{N}, \\
n & =\mathrm{e}^{-4 U}\left(\dot{a}+\mathbf{Z}^{T} \mathbb{C} \dot{\mathbf{Z}}\right),
\end{aligned}
$$

where we organised the electric and magnetic charges in the symplectic vector

$$
\mathcal{Q}^{N}=\left(m^{I}, e_{I}\right)
$$

The mass of the black hole spacetime is defined by the Komar integral

$$
M=-\frac{1}{8 \pi} \int_{S_{\infty}^{2}} \nabla^{\alpha} \xi^{\beta} \mathrm{d} S_{\alpha \beta}, \quad \mathrm{d} S_{\alpha \beta}=-2 n_{[\alpha} r_{\beta]} \sqrt{\sigma} \mathrm{d} \theta \mathrm{d} \phi,
$$

where $\xi^{\alpha}=\xi^{t}=1$ is the timelike killing vector, $n^{\alpha}=n^{t}=\mathrm{e}^{-U}$ is the timelike normal to $S_{\infty}^{2}, r^{\beta}=r^{\tau}=\mathrm{e}^{U-2 A}$ is the spacelike (radial) normal to $S_{\infty}^{2}$, and finally, $\sqrt{\sigma}=\sin \theta \mathrm{e}^{2 A-2 U}$ is the determinant of the induced metric on $S_{\infty}^{2}$. We then find

$$
M=\Gamma_{t \tau}^{t}(0)=\dot{U}(0) .
$$

Open Access. This article is distributed under the terms of the Creative Commons Attribution Noncommercial License which permits any noncommercial use, distribution, and reproduction in any medium, provided the original author(s) and source are credited.

\section{References}

[1] T. Mohaupt, Black holes in supergravity and string theory, Class. Quant. Grav. 17 (2000) 3429 [hep-th/0004098] [SPIRES].

[2] L. Andrianopoli, R. D'Auria, S. Ferrara and M. Trigiante, Extremal black holes in supergravity, Lect. Notes Phys. 737 (2008) 661 [hep-th/0611345] [SPIRES].

[3] I. Bena and N.P. Warner, Black holes, black rings and their microstates, Lect. Notes Phys. 755 (2008) 1 [hep-th/0701216] [SPIRES].

[4] S. Ferrara, K. Hayakawa and A. Marrani, Lectures on attractors and black holes, Fortsch. Phys. 56 (2008) 993 [arXiv:0805.2498] [SPIRES].

[5] S. Bellucci, S. Ferrara, M. Günaydin and A. Marrani, SAM lectures on extremal black holes in $D=4$ extended supergravity, arXiv:0905.3739 [SPIRES].

[6] G.W. Gibbons, R. Kallosh and B. Kol, Moduli, scalar charges and the first law of black hole thermodynamics, Phys. Rev. Lett. 77 (1996) 4992 [hep-th/9607108] [SPIRES].

\footnotetext{
${ }^{17}$ The killing one-form $e_{t}=\mathrm{d} t+A_{K} K$ is not hyper surface orthogonal since it is not closed $\mathrm{d} e_{t}=F_{K K} \neq 0$. Hence we cannot define a spatial hypersurface orthogonal to the Killing vector.
} 
[7] S. Ferrara, G.W. Gibbons and R. Kallosh, Black holes and critical points in moduli space, Nucl. Phys. B 500 (1997) 75 [hep-th/9702103] [SPIRES].

[8] L. Andrianopoli, R. D'Auria, E. Orazi and M. Trigiante, First order description of D=4 static black holes and the Hamilton-Jacobi equation, Nucl. Phys. B 833 (2010) 1 [arXiv:0905.3938] [SPIRES].

[9] A. Ceresole and G. Dall'Agata, Flow equations for non-BPS extremal black holes, JHEP 03 (2007) 110 [hep-th/0702088] [SPIRES].

[10] G. Lopes Cardoso, A. Ceresole, G. Dall'Agata, J.M. Oberreuter and J. Perz, First-order flow equations for extremal black holes in very special geometry, JHEP 10 (2007) 063 [arXiv:0706.3373] [SPIRES].

[11] L. Andrianopoli, R. D'Auria, E. Orazi and M. Trigiante, First order description of black holes in moduli space, JHEP 11 (2007) 032 [arXiv:0706.0712] [SPIRES].

[12] S. Ferrara, A. Marrani and E. Orazi, Maurer-Cartan equations and black hole superpotentials in $N=8$ supergravity, Phys. Rev. D 81 (2010) 085013 [arXiv:0911.0135] [SPIRES].

[13] G. Bossard, Y. Michel and B. Pioline, Extremal black holes, nilpotent orbits and the true fake superpotential, JHEP 01 (2010) 038 [arXiv:0908.1742] [SPIRES].

[14] A. Ceresole, G. Dall'Agata, S. Ferrara and A. Yeranyan, Universality of the superpotential for $D=4$ extremal black holes, arXiv:0910.2697 [SPIRES].

[15] A. Ceresole, G. Dall'Agata, S. Ferrara and A. Yeranyan, First order flows for $N=2$ extremal black holes and duality invariants, Nucl. Phys. B 824 (2010) 239 [arXiv:0908.1110] [SPIRES].

[16] C.M. Miller, K. Schalm and E.J. Weinberg, Nonextremal black holes are BPS, Phys. Rev. D 76 (2007) 044001 [hep-th/0612308] [SPIRES].

[17] G.L. Cardoso and V. Grass, On five-dimensional non-extremal charged black holes and FRW cosmology, Nucl. Phys. B 803 (2008) 209 [arXiv:0803.2819] [SPIRES].

[18] B. Janssen, P. Smyth, T. Van Riet and B. Vercnocke, A first-order formalism for timelike and spacelike brane solutions, JHEP 04 (2008) 007 [arXiv:0712.2808] [SPIRES].

[19] J. Perz, P. Smyth, T. Van Riet and B. Vercnocke, First-order flow equations for extremal and non-extremal black holes, JHEP 03 (2009) 150 [arXiv:0810.1528] [SPIRES].

[20] P. Breitenlohner, D. Maison and G.W. Gibbons, Four-dimensional black holes from Kaluza-Klein theories, Commun. Math. Phys. 120 (1988) 295 [SPIRES].

[21] E. Cremmer et al., Euclidean-signature supergravities, dualities and instantons, Nucl. Phys. B 534 (1998) 40 [hep-th/9803259] [SPIRES].

[22] V. Cortes, C. Mayer, T. Mohaupt and F. Saueressig, Special geometry of euclidean supersymmetry. I: vector multiplets, JHEP 03 (2004) 028 [hep-th/0312001] [SPIRES].

[23] C.M. Hull and B. Julia, Duality and moduli spaces for time-like reductions, Nucl. Phys. B 534 (1998) 250 [hep-th/9803239] [SPIRES].

[24] V. Cortes and T. Mohaupt, Special geometry of euclidean supersymmetry III: the local r-map, instantons and black holes, JHEP 07 (2009) 066 [arXiv:0905.2844] [SPIRES].

[25] E. Bergshoeff, W. Chemissany, A. Ploegh, M. Trigiante and T. Van Riet, Generating geodesic flows and supergravity solutions, Nucl. Phys. B 812 (2009) 343 [arXiv:0806.2310] [SPIRES]. 
[26] D.V. Gal'tsov and O.A. Rytchkov, Generating branes via $\sigma$-models, Phys. Rev. D 58 (1998) 122001 [hep-th/9801160] [SPIRES].

[27] M. Günaydin, A. Neitzke, B. Pioline and A. Waldron, Quantum attractor flows, JHEP 09 (2007) 056 [arXiv:0707.0267] [SPIRES].

[28] P. Dobiasch and D. Maison, Stationary, spherically symmetric solutions of Jordan's unified theory of gravity and electromagnetism, Gen. Rel. Grav. 14 (1982) 231 [SPIRES].

[29] G. Bossard, H. Nicolai and K.S. Stelle, Universal BPS structure of stationary supergravity solutions, JHEP 07 (2009) 003 [arXiv: 0902 .4438] [SPIRES].

[30] G. Bossard, The extremal black holes of $N=4$ supergravity from $\mathrm{SO}(8,2+n)$ nilpotent orbits, Gen. Rel. Grav. 42 (2010) 539 [arXiv:0906.1988] [SPIRES].

[31] G. Bossard, Extremal black holes and nilpotent orbits, arXiv:0910.0689 [SPIRES].

[32] G. Bossard, 1/8 BPS black hole composites, arXiv:1001.3157 [SPIRES].

[33] D. Gaiotto, W.W. Li and M. Padi, Non-supersymmetric attractor flow in symmetric spaces, JHEP 12 (2007) 093 [arXiv:0710.1638] [SPIRES].

[34] P. Fré et al., Cosmological backgrounds of superstring theory and solvable algebras: oxidation and branes, Nucl. Phys. B 685 (2004) 3 [hep-th/0309237] [SPIRES].

[35] P. Fré' and A.S. Sorin, The arrow of time and the Weyl group: all supergravity billiards are integrable, arXiv:0710.1059 [SPIRES].

[36] P. Fré and A.S. Sorin, The Weyl group and asymptotics: all supergravity billiards have a closed form general integral, Nucl. Phys. B 815 (2009) 430 [SPIRES].

[37] P. Fré and A.S. Sorin, The integration algorithm for nilpotent orbits of $G / H^{*}$ Lax systems: for extremal black holes, arXiv:0903.3771 [SPIRES].

[38] M. Berkooz and B. Pioline, 5d black holes and non-linear $\sigma$-models, JHEP 05 (2008) 045 [arXiv:0802.1659] [SPIRES].

[39] W. Chemissany, A. Ploegh and T. Van Riet, A note on scaling cosmologies, geodesic motion and pseudo-SUSY, Class. Quant. Grav. 24 (2007) 4679 [arXiv:0704.1653] [SPIRES].

[40] W. Chemissany, P. Fré and A.S. Sorin, The integration algorithm of Lax equation for both generic Lax matrices and generic initial conditions, Nucl. Phys. B $\mathbf{8 3 3}$ (2010) 220 [arXiv: 0904.0801] [SPIRES].

[41] W. Chemissany, J. Rosseel, M. Trigiante and T. Van Riet, The full integration of black hole solutions to symmetric supergravity theories, Nucl. Phys. B 830 (2010) 391 [arXiv: 0903.2777] [SPIRES].

[42] P. Fré and J. Rosseel, On full-fledged supergravity cosmologies and their Weyl group asymptotics, arXiv:0805.4339 [SPIRES].

[43] P. Fré and A.S. Sorin, Integrability of supergravity billiards and the generalized Toda lattice equation, Nucl. Phys. B 733 (2006) 334 [hep-th/0510156] [SPIRES].

[44] M. Günaydin, Lectures on spectrum generating symmetries and U-duality in supergravity, extremal black holes, quantum attractors and harmonic superspace, arXiv:0908.0374 [SPIRES].

[45] P. Figueras, E. Jamsin, J.V. Rocha and A. Virmani, Integrability of five dimensional minimal supergravity and charged rotating black holes, Class. Quant. Grav. 27 (2010) 135011 [arXiv: 0912.3199] [SPIRES]. 
[46] S.-S. Kim, J.L. Hornlund, J. Palmkvist and A. Virmani, Extremal solutions of the S3 model and nilpotent orbits of G2(2), JHEP 08 (2010) 072 [arXiv: 1004.5242] [SPIRES].

[47] T. Mohaupt and O. Vaughan, Non-extremal black holes, harmonic functions and attractor equations, arXiv:1006.3439 [SPIRES].

[48] P. Fré and A.S. Sorin, Supergravity black holes and billiards and Liouville integrable structure of dual Borel algebras, JHEP 03 (2010) 066 [arXiv:0903.2559] [SPIRES].

[49] O. Babelon and C.M. Viallet, Hamiltonian structures and Lax equations, Phys. Lett. B 237 (1990) 411 [SPIRES].

[50] P. Fre, A.S. Sorin and M. Trigiante, work in progress.

[51] L. Andrianopoli, R. D'Auria, S. Ferrara and M. Trigiante, Fake superpotential for large and small extremal black holes, JHEP 08 (2010) 126 [arXiv: 1002.4340] [SPIRES].

[52] J. de Boer, E.P. Verlinde and H.L. Verlinde, On the holographic renormalization group, JHEP 08 (2000) 003 [hep-th/9912012] [SPIRES].

[53] K. Hotta, Holographic RG flow dual to attractor flow in extremal black holes, Phys. Rev. D 79 (2009) 104018 [arXiv:0902.3529] [SPIRES].

[54] Y. Kodama and J. Ye, Iso-spectral deformations of general matrix and their reductions on Lie algebras, solv-int/9506005.

[55] Y. Kodama and M. K. T.-R., Explicit integration of the full symmetric Toda hierarchy and the sorting property, solv-int/9502006.

[56] Y. Kodama and J. Ye, Toda hierarchy with indefinite metric, solv-int/9505004.

[57] L.A. Ferreira and D.I. Olive, Noncompact symmetric spaces and the Toda molecule equations, Commun. Math. Phys. 99 (1985) 365 [SPIRES].

[58] V. Arnold, Mathematical methods of classical mechanics, Springer, U.S.A. (1997).

[59] J. McCauley, Classical mechanics: transformations, flows, integrable and chaotic dynamics, Cambridge University Press, Cambridge U.K. (1997).

[60] K.R. Meyer, G.R. Hall and D. Offin, Introduction to hamiltonian dynamical systems and the N-body problem, Springer, U.S.A. (2009).

[61] G.W. Gibbons, Antigravitating black hole solitons with scalar hair in $N=4$ supergravity, Nucl. Phys. B 207 (1982) 337 [SPIRES].

[62] L. Andrianopoli, R. D'Auria, S. Ferrara, P. Fré and M. Trigiante, $R$ - $R$ scalars, U-duality and solvable Lie algebras, Nucl. Phys. B 496 (1997) 617 [hep-th/9611014] [SPIRES]. 